\title{
Investigation into the rheology of a solid sphere suspension in second-order fluid using a cell model
}

\author{
Liam J. Escott $\odot^{*}$ and Helen J. Wilson $(\odot$ \\ Department of Mathematics, UCL, Gower Street, London WC1E 6BT, United Kingdom
}

(Received 28 June 2019; accepted 26 June 2020; published 3 August 2020)

\begin{abstract}
We consider a suspension of solid spheres in a viscoelastic suspending fluid. We use the second-order fluid model to capture the first effects of viscoelasticity in the suspension and a mean-field cell model to express the influence of the solid particles. We create a semianalytical constitutive equation for the whole suspension, a second-order fluid with modified material parameters. We find that, in simple shear, the ratio of first normal stress difference to shear stress is independent of volume fraction, but that the second normal stress difference can change sign as the volume fraction of solids increases. Our model is valid for all flows for which the second-order fluid expansion is applicable.
\end{abstract}

DOI: 10.1103/PhysRevFluids.5.083301

\section{INTRODUCTION}

Suspensions of particles in a fluid matrix are ubiquitous in nature and industry, from blood to mud and from paint to slurries. There is a real need for simple constitutive models for these systems, and their study goes back over a century.

Particle suspensions, even in Newtonian fluid matrices, can show both shear thinning and shear thickening (e.g., Ref. [1]). Shear thickening is associated with jamming [2] and has been the focus of much recent theoretical and simulation work (e.g., Refs. [3-5]). The current consensus is that either friction, or something resembling friction, between particles is required for strong discontinuous shear thickening. Shear-thinning, however, can occur through breakdown of weakly attracted colloidal clusters [6] or by the exposure of the supposedly Newtonian matrix fluid to extreme local shear rates at which it itself is shear-thinning [7].

Once the matrix fluid is explicitly non-Newtonian, the behavior of the system becomes even more complex. The next level of complexity is generalised Newtonian fluids-matrix fluids which can be described in terms of a single scalar viscosity, whose value may depend on the local shear rate. Early experiments by Highgate and Whorlow [8] using dilute suspensions in a shear-thinning matrix with no viscoelastic effects found that the viscosity of the suspension could be captured well using an Einstein-like form as long as $\eta_{0}$, the viscosity of the matrix fluid, was chosen at the appropriate shear stress. Many subsequent papers (e.g., Refs. [9-11]) have confirmed the existence of scaling laws relating the rheology of the suspension to that of the suspending medium.

When viscoelasticity is added, the suspensions exhibit normal stresses in shear flow. At moderate concentrations, the first normal stress difference $N_{1}$ is observed to be positive, whereas the second normal stress difference $N_{2}$ is negative; and the magnitude of both increases with the volume fraction of particles [12-15].

Recent theoretical work for dilute suspensions [16] has shown that suspensions in a polymeric suspending fluid can shear-thicken even when the fluid's shear viscosity is constant, due to streamwise stretching of the polymers. Rapid progress is also being made on simulating suspensions

*liam.escott.12@ucl.ac.uk 
in a polymeric matrix fluid [17]. However, simulations, while exceedingly useful, do not have the capability to yield a short, usable constitutive model that can be use as a rule of thumb, or as an ingredient in larger-scale CFD simulations.

In this paper we will focus on the analytical modeling of suspension rheology. Research in this area began with Einstein [18], who calculated the leading-order correction to the viscosity of a Newtonian fluid of viscosity $\eta_{0}$ when solid spheres are added at dilute volume fraction $\phi$ :

$$
\eta_{\mathrm{eff}}=\eta_{0}(1+5 \phi / 2) \text {. }
$$

Because of its simplicity and useability, the "Einstein viscosity" (as it has become known) is widely used in many areas, often as a first correction rather than a full constitutive model.

Many authors have attempted to extend this model. For Newtonian fluid matrices, where the task is to extend the model to higher solids volume fractions, the only really effective analytical work is by Batchelor and Green [19], who extended Einstein's analysis to interacting pairs of spheres. They found that the system could still be represented by a single scalar viscosity for each steady flow type; but that this number was indeterminate for some flows (including shear flow) which admit the possiblity of orbiting particles. Where the viscosity is well-defined, they calculated it in terms of hydrodynamic functions $J(s)$ and $q(s)$ as

$$
\eta_{\mathrm{eff}}=\eta_{0}\left[1+\frac{5}{2} \phi+\left(\frac{5}{2}+\int_{2}^{\infty} J(s) q(s) \mathrm{d} s\right) \phi^{2}\right] \approx \eta_{0}\left(1+\frac{5}{2} \phi+6.9 \phi^{2}\right) .
$$

However, while the Einstein viscosity provides a reasonable approximation to experimentally observed viscosities for volume fractions up to $10 \%$, the addition of the Batchelor and Green correction term does not extend the range of validity very far before terms of order $\phi^{3}$ and higher become important-and these terms are intractable to calculate analytically.

We then move to empirical models. There are many of these, which use a variety of physical arguments to capture the viscosity divergence close to maximum packing (e.g., Refs. [20,21]). The most commonly used empirical model, largely based on its simplicity, is the Krieger-Dougherty model [22]:

$$
\eta_{\mathrm{eff}}=\eta_{0}\left(1-\frac{\phi}{\phi_{m}}\right)^{-5 \phi_{m} / 2}
$$

an empirical model which mimics Eq. (1) for small $\phi$ and diverges as $\phi \rightarrow \phi_{m}$, the maximum packing fraction. The parameter $\phi_{m}$ depends on the particle shape and on the details of interactions between close particles; it is often taken to be around $\phi_{m}=0.64$.

However, another important class of suspending matrices is viscoelastic fluids, and here the theoretical work is much less well developed. All viscoelastic fluids are reducible, in the slow flow limit, to the second-order fluid, so that is a natural place to start. There have been several erroneous attempts to extend the Einstein viscosity to second-order fluids [23-28]; the correct analysis was carried out by Koch and Subramanian [29], yielding the full steady rheology in terms of the properties $\eta_{0}$, first normal stress difference coefficient $\Psi_{1}=N_{1} / \dot{\gamma}^{2}$ and second normal stress difference coefficient $\Psi_{2}=N_{2} / \dot{\gamma}^{2}$ of the suspending fluid:

$$
\eta_{\text {eff }}=\eta_{0}\left(1+\frac{5}{2} \phi\right), \quad \Psi_{1, \text { eff }}=\Psi_{1}\left(1+\frac{5}{2} \phi\right), \quad \Psi_{2, \text { eff }}=\Psi_{2}\left(1+\frac{75}{28} \phi\right)+\Psi_{1} \frac{5}{56} \phi .
$$

The discrepancy between Koch and Subramanian [30] and the two later papers [27,28] was finally resolved by Rallison [31], who showed that a divergent integral inherent in the volume-average method had been neglected in both the later papers and confirmed that the ensemble-average method used by Ref. [30] was correct.

In this paper we focus primarily on the dependence of fluid rheology on particle concentration. We will generate a simple constitutive model—a second-order fluid itself-for concentrated suspensions in a second-order background fluid. 


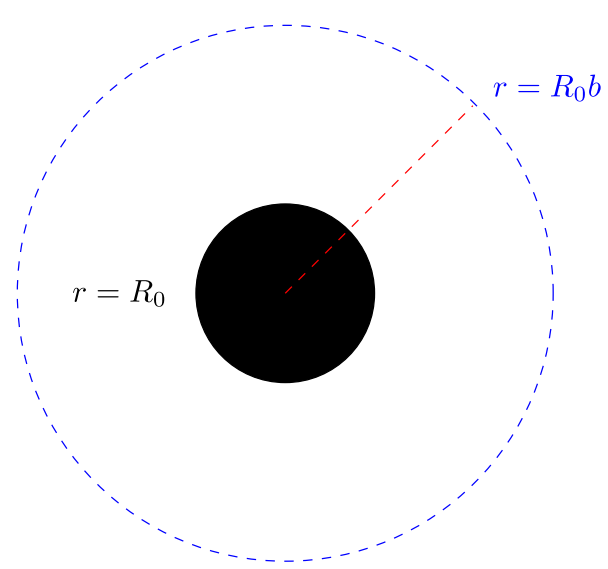

FIG. 1. Schematic of the cell model. The outer boundary is a virtual surface outside which the flow is unaffected by the presence of the solid sphere. It remains fixed relative to the particle and does not deform with the flow.

In Sec. II we introduce the cell model around which our analysis is based, and discuss our governing equations, boundary conditions and solution strategy. In Sec. III we solve the Newtonian flow problem and verify against existing literature; we are then able to propose an empirical "fix" for the cell model to correct its viscosity predictions. In Sec. IV we solve the full second-order fluid system and give quantitative predictions for the rheology of a suspension in a second-order fluid matrix. Finally, we present our conclusions in Sec. V.

\section{THE CELL MODEL}

\section{A. Introduction}

In this paper we will use the cell model approach to produce a practical, semiempirical model of the effect of solid spherical particles on the rheology of a second-order fluid. We consider a suspension of identical, force-free, torque-free, spherical particles, each of radius $R_{0}$, at solids volume fraction $\phi$. The key concept of the model is to allocate to each particle a "sphere of influence," as depicted schematically in Fig. 1, outside which the flow is unaffected by the presence of the particle. We refer to this restricted volume as the "cellular fluid phase." The outer sphere is not a material surface but provides a conceptual boundary, across which the nature of the flow may change. Each cell is then considered to be suspended in a medium which has the same rheology as the macroscopic suspension.

The cell model concept was first introduced by Simha [32] and Happel [33], using a Newtonian background fluid, and it is still widely used in more complex situations for which experiments or simulations may be more difficult (see, for example, [34-37]). Mifflin [25] first attempted to apply the cell model to a non-Newtonian suspending fluid. However, the attempt to find the dilute result with this model was unsuccessful due to an ill defined integral for dissipation, which we discuss further in Sec. II D. These models in their standard form are not expected to be accurate for very concentrated suspensions: typically the model viscosity diverges as $\phi \rightarrow 1$, where in reality flow will cease around $\phi=0.64$ and the maximum close packing fraction for identical spheres is $\phi=0.74$. However, when solved correctly for Newtonian fluids, these models do reduce to the Einstein viscosity in the dilute limit.

The cell model is essentially a mean-field approximation: Each particle feels the influence of the rest of the suspension only as an average, rather than sampling the details of close interactions with neighboring particles. As such, it can never be fully accurate at high concentration; however, 
as mentioned above, we will propose an empirical modification in Sec. III which can improve its performance.

There is numerical evidence to suggest that the cell model is a valuable technique in calculating viscometric functions in suspension. Yang and Shaqfeh [38] investigated the mechanism of shear thickening in dilute suspensions, using a conformation tensor $C_{i j}$ to describe three models for the polymer stress: Oldroyd-B, Giesekus, and FENE-P. For the Oldroyd-B model, isocontours of $C_{k k}$ at $10 \%$ of maximum value were plotted about a particle, and the length of the wake used to define a spherical volume, akin to the sphere of influence in our investigation. The percentage of all viscometric functions $C_{12}, C_{11}-C_{22}$, and $C_{22}-C_{33}$, which are captured in this region close to the particle surface were calculated and presented for $\mathrm{O}(1)$ values of Weissenberg number Wi. The results show that a large percentage of the viscometric functions come from this volume close to the particle, and also that this percentage stays constant with respect to Wi. This numerical analysis suggests to us that it is likely valid, at least in the dilute region, to apply a cell model around particles in suspension.

Einarsson et al. [39] analytically investigated the rheology of a dilute suspension in an Oldroyd-B background fluid to $O\left(\mathrm{Wi}^{2}\right)$. They identified the contributions to velocity gradient about one particle in an infinite fluid phase, and plotted this using an eigenvalue technique introduced by Chong et al. [40]. They found that the shear thickening mechanism in their system was due to the "particle induced fluid stress in regions of flow around the particle with a strong straining component." These regions are given the name "hot spots" in Ref. [39], and have a key identifying factor of being near to the particle surface. Outside a certain radius from the center of the solid particle, these regions cease to make a contribution to the viscometric functions of the fluid matrix which, like the work above, suggests to us that the rheology can be captured by considering a cell model about each particle in suspension. This analysis was completed under the assumption of low solid volume fraction, and we will investigate how applicable the cell model is at larger concentrations.

There is some debate as to which boundary conditions to apply on the cell surface: a longstanding discrepancy between the results of Simha [32] and Happel [33] on this issue has recently been clarified by Sherwood [41]. He discusses the options used in the early literature: continuity of velocity [32] or continuity of tangential stress and normal velocity [33]. Of the two, continuity of velocity has certain advantages: in particular, if the velocity is imposed on the outer surface of the cell then the volume-averaged strain rate within the entire cell (including the solid region) is automatically equal to $\mathbf{E}^{\infty}$, which is not true of the stress-continuity case. Continuity of velocity will therefore be our choice.

\section{B. Second-order fluid}

We assume that the fluid is incompressible and that inertia may be neglected. Then the governing equations for steady flow with velocity field $\mathbf{v}$ are mass conservation:

$$
\nabla \cdot \mathbf{v}=0
$$

and momentum balance:

$$
\nabla \cdot \sigma=\mathbf{0}
$$

in which the stress tensor $\sigma$ for a second-order fluid is given by

$$
\boldsymbol{\sigma}=-p \mathbf{I}+2 \eta_{0} \mathbf{E}+\alpha_{0} \mathbf{A}+4 \alpha_{1} \mathbf{E} \cdot \mathbf{E} .
$$

Here $p$ is the pressure, $E_{i j}$ the rate of strain tensor, and $A_{i j}$ is the second Rivlin-Ericksen tensor [42], defined using the lower-convected derivative. Specifically, in terms of the velocity gradient

$$
L_{i j}=(\nabla \mathbf{v})_{i j}=\nabla_{j} v_{i},
$$

we define

$$
\mathbf{E}=\frac{1}{2}\left(\mathbf{L}+\mathbf{L}^{\top}\right)
$$


and, for steady flows,

$$
\mathbf{A}=2\left(\mathbf{v} \cdot \nabla \mathbf{E}+\mathbf{L}^{\top} \cdot \mathbf{E}+\mathbf{E} \cdot \mathbf{L}\right)
$$

The special case $\alpha_{0}=\alpha_{1}=0$ gives us a Newtonian fluid of viscosity $\eta_{0}$, for which Eqs. (6) and (7) combine to give the Stokes equation:

$$
\nabla p=\eta_{0} \nabla^{2} \mathbf{v}
$$

Lengths scale with the particle radius $R_{0}$, velocities with some typical velocity scale $U$, and stresses and pressures with a typical Newtonian shear stress $\eta_{0} U / R_{0}$, these governing equations become (with dimensionless quantities denoted with a star):

$$
\sigma_{i j}^{*}=-p^{*} \delta_{i j}+2 E_{i j}^{*}+\mathrm{Wi}\left(\epsilon A_{i j}^{*}+4 E_{i k}^{*} E_{k j}^{*}\right)
$$

in which we have introduced two dimensionless parameters: the Weissenberg number Wi $=$ $\alpha_{1} U / \eta_{0} R_{0}$ (the ratio of elastic to viscous forces) and $\epsilon=\alpha_{0} / \alpha_{1}$, a material parameter of the second-order fluid model. We have chosen $\alpha_{0}$ for our definition of Wi, but some others use $\alpha_{1}$, so care needs to be taken in comparing results in this area.

Suppressing stars henceforth, our governing momentum equation becomes

$$
-\nabla p+\nabla^{2} \mathbf{v}+\operatorname{Wi} \nabla \cdot(\epsilon \mathbf{A}+4 \mathbf{E} \cdot \mathbf{E})=\mathbf{0} .
$$

\section{Boundary conditions}

We apply a background flow outside the cell which is linear in space:

$$
\mathbf{u}^{\infty}=\mathbf{U}^{\infty}+\boldsymbol{\Omega}^{\infty} \cdot \mathbf{x}+\mathbf{E}^{\infty} \cdot \mathbf{x}
$$

in which $\boldsymbol{\Omega}^{\infty}$ is an antisymmetric tensor describing rotation, and $\mathbf{E}^{\infty}$ a symmetric tensor capturing strain. We consider the case of force-free, torque-free spheres, which translate and rotate passively with the fluid around them: the solid sphere is therefore in rigid-body motion,

$$
\mathbf{u}^{\text {solid }}=\mathbf{U}^{\infty}+\boldsymbol{\Omega}^{\infty} \cdot \mathbf{x}
$$

For brevity, we choose to change our frame of reference to one translating with the particle at velocity $\mathbf{U}^{\infty}$. This leaves us with a simpler far-field flow:

$$
\mathbf{u}^{\infty}=\boldsymbol{\Omega}^{\infty} \cdot \mathbf{x}+\mathbf{E}^{\infty} \cdot \mathbf{x},
$$

and a boundary condition on the surface of the solid sphere,

$$
\mathbf{v}=\boldsymbol{\Omega}^{\infty} \cdot \mathbf{x} \text { on } r=1 .
$$

Finally, velocity continuity at the outer surface of the cell gives

$$
\mathbf{v}=\boldsymbol{\Omega}^{\infty} \cdot \mathbf{x}+\mathbf{E}^{\infty} \cdot \mathbf{x} \text { on } r=b .
$$

\section{Averaging technique}

To calculate the effective rheology of the suspension, we need to find the bulk, or average, extra stress generated by the particles. It was shown by Rallison [31] that a volume average [as applied by Ref. 27, for example] is not suitable for use with a second-order fluid in the limit $b \rightarrow \infty$, as it generates a divergent integral [neglected by Ref. 27]. Instead we follow the technique introduced by Koch and Subramanian [30] and use an ensemble average. We first average over all possible configurations of particles in the suspension, and then apply the fluid cell model. This introduces the dependence of cell radius $b$ into certain average terms which appear in the calculation of bulk stress. For finite cell volume, the results are equivalent to a volume average calculation, but in the limit $b \rightarrow \infty$ our results can be verified against those of Koch and Subramanian [30] but disagree with Greco et al. [27]. 
We will calculate the ensemble average of the deviatoric stress tensor over the whole suspension. We have to average the stress over both the solid and fluid phase, which requires an equation for the stress inside the particles. Rigorously therefore, we define $\sigma_{i j}^{P}$ as the extra stress within the solid phase if the fluid constitutive equations applied within the particle:

$$
\sigma_{i j}^{P}=\sigma_{i j}-\left[-p \delta_{i j}+2 E_{i j}+\operatorname{Wi}\left(\epsilon A_{i j}+4 E_{i k} E_{k j}\right)\right] \text { for } r \leqslant 1 .
$$

In this way we may describe the stress within the entire suspension as the fluid stress with an extra stress term valid only within the solid phase. Suppose that we have found the steady solution to Eqs. (5) and (12) which satisfies our boundary conditions Eqs. (16) and (17). Then, denoting by $\widehat{\mathbf{Q}}$ the deviatoric part of tensor $\mathbf{Q}$, and the ensemble average of quantity $Q$ by $\langle Q\rangle$, we have

$$
\langle\widehat{\boldsymbol{\sigma}}\rangle=2\langle\mathbf{E}\rangle+\operatorname{Wi}(\epsilon\langle\widehat{\mathbf{A}}\rangle+4\langle\widehat{\mathbf{E} \cdot \mathbf{E}}\rangle)+N \int_{V^{1}} \widehat{\left\langle\boldsymbol{\sigma}^{P}\right\rangle} d V,
$$

with $V^{1}$ the volume of a single sphere of unit radius. The first two terms come directly from Eq. (11), noting that the pressure contribution disappears from the deviatoric stress because it is isotropic. We also used here the particle number density $N$, defined as the number of particles per unit volume of suspension: however, since each particle has dimensionless volume $4 \pi / 3$ we will write $N=3 \phi / 4 \pi$.

All terms in Eq. (19) require some care due to the step change in $\mathbf{E}$ at the particle surface. Within the fluid phase the rate of strain is calculated directly from the local velocity field, whereas in the solid phase it is identically zero. Therefore, it is necessary to show that no terms provide a singular contribution to the averaged stress tensor. Koch and Subramanian [30] investigated this step change, and managed to prove analytically that the mean polymer stress is both zero within the solid phase and nonsingular at the surface. The net result is that neither of the first two terms in Eq. (19) provide ill-posed averages and $\sigma^{P}$ differs from $\sigma$ only by an isotropic term.

The deviatoric stress tensor is therefore sufficient to determine the macroscopic rheology of the suspension as a whole. After use of the divergence theorem, our expression for the ensemble averaged deviatoric stress reduces to

$$
\langle\widehat{\boldsymbol{\sigma}}\rangle=2\langle\mathbf{E}\rangle+\operatorname{Wi}(\epsilon\langle\widehat{\mathbf{A}}\rangle+4\langle\widehat{\mathbf{E} \cdot \mathbf{E}}\rangle)+N \widehat{\mathbf{S}},
$$

in which $\mathbf{S}$ is the particle stresslet, defined as

$$
S_{i j}=\frac{1}{2} \int_{r=1}\left(\left\langle\sigma_{i k}\right\rangle \hat{n}_{j}+\left\langle\sigma_{k j}\right\rangle \hat{n}_{i}\right) \hat{n}_{k} d S,
$$

where $\hat{\mathbf{n}}$ is the outer unit normal to the particle. Since the cell model isolates each particle from all others, the average stress at the particle surface appearing in Eq. (21) is exactly the stress found by solving the fluid governing equations, evaluated at $r=1$.

The term $\langle\mathbf{E}\rangle$ in (20) is well-behaved in the limit $b \rightarrow \infty$, so in this case we can replace the ensemble average by the volume average. The volume average is guaranteed, by the use of velocity continuity boundary conditions at the outside of the cell, to return the far-field rate of strain $\mathbf{E}^{\infty}$. The same is true of the antisymmetric part of the rate of strain tensor $\boldsymbol{\Omega}=\frac{1}{2}\left(\mathbf{L}-\mathbf{L}^{\top}\right)$, whose volume average is $\boldsymbol{\Omega}^{\infty}$.

To evaluate the remaining averages $\langle\widehat{\mathbf{A}}\rangle$ and $\langle\widehat{\mathbf{E} \cdot \mathbf{E}}\rangle$, which would diverge in the limit $b \rightarrow \infty$ if we attempted to use a volume integral, we write the local variables $\mathbf{v}, \mathbf{E}$ and $\boldsymbol{\Omega}$ in terms of their mean values and the deviation from those mean values:

$$
v_{i}=v_{i}^{\infty}+v_{i}^{\prime} ; \quad E_{i j}=E_{i j}^{\infty}+E_{i j}^{\prime} ; \quad \Omega_{i j}=\Omega_{i j}^{\infty}+\Omega_{i j}^{\prime} .
$$

Writing

$$
A_{i j}=2\left[\nabla_{k}\left(v_{k} E_{i j}\right)+\Omega_{k i} E_{k j}+E_{i k} \Omega_{k j}+2 E_{i k} E_{k j}\right],
$$


and noting that $\left\langle v_{i}^{\prime}\right\rangle=\left\langle E_{i j}^{\prime}\right\rangle=\left\langle\Omega_{i j}^{\prime}\right\rangle=0$, we can rewrite the mean polymer stress term as

$$
\begin{aligned}
\epsilon\left\langle\widehat{A_{i j}}\right\rangle+4\left\langle\widehat{E_{i k} E_{k j}}\right\rangle & =2 \epsilon\left[\left\langle\nabla_{k}\left(v_{k} E_{i j}\right)\right\rangle+\left\langle\widehat{\Omega_{k i} E_{k j}}\right\rangle+\left\langle\widehat{E_{i k} \Omega_{k j}}\right\rangle\right]+4(1+\epsilon)\left\langle\widehat{E_{i k} E_{k j}}\right\rangle \\
& =4 \epsilon\left\langle\widehat{E_{i k}^{\prime} \Omega_{k j}^{\prime}}\right\rangle+4 \epsilon \widehat{E_{i k}^{\infty} \Omega_{k j}^{\infty}}+4(1+\epsilon)\left\langle\widehat{E_{i k}^{\prime} E_{k j}^{\prime}}\right\rangle+4(1+\epsilon) \widehat{E_{i k}^{\infty} E_{k j}^{\infty}} .
\end{aligned}
$$

The convective term disappears under averaging since the gradient operator and ensemble average commute, and the term $\left\langle v_{k} E_{i j}\right\rangle$ is independent of position. We note also that Eq. (24) is a summation of the mean polymer stress without solid phase and the extra polymer stress contribution from the particles, expressed as the two ensemble averages remaining. These added terms can be reduced to integrals over the cellular fluid volume [30]:

$$
\left\langle E_{i k}^{\prime} \Omega_{k j}^{\prime}\right\rangle=\frac{3 \phi}{4 \pi} \int_{V^{b}} E_{i k}^{\prime} \Omega_{k j}^{\prime} d V ; \quad\left\langle E_{i k}^{\prime} E_{k j}^{\prime}\right\rangle=\frac{3 \phi}{4 \pi} \int_{V^{b}} E_{i k}^{\prime} E_{k j}^{\prime} d V .
$$

Herein lies the difference between our use of the cell model and that of Mifflin [25]. While our analyses up until the averaging process have been the same, instead of taking an ensemble average over the suspension, [25] considered a dissipation integral $D$ given by

$$
D=\int_{V^{b}-V^{1}} E_{i j}\left(\epsilon A_{i j}+4 E_{i k} E_{k j}\right) d V .
$$

The issue, as first shown by Koch and Subramanian [30] is that at the sphere of influence $r=b$, there is a step change in the strain rate from the calculated $E_{i j}$ inside the cell volume to $E_{i j}^{\infty}$ forced by the boundary conditions. This causes an $O(b)$ contribution to the integral from the convection term in $A_{i j}$, which diverges in the dilute limit. Our method avoids this problem in two distinct ways, firstly the ensemble averaging process as shown above guarantees that we need not consider any integral over that convection term. Second, we take an average directly over the stress of the suspension, and therefore need not consider the contraction of the stress with the rate of strain tensor, as needed in Eq. (26).

\section{E. Extension of the cell model}

Previous authors who have worked with the cell model have assumed that each fluid element in the system is contained within exactly one cell: thus, there is a direct relationship between the solids volume fraction $\phi$ and the dimensionless cell size, $b$, namely,

$$
\phi=b^{-3} \text {. }
$$

While this is clearly appropriate in the dilute limit (for which the cell model with a Newtonian solvent fluid reproduces the Einstein viscosity), we argue that this assumption should be relaxed when considering more concentrated systems.

Consider the dense system sketched in Fig. 2. We now use the sphere of influence to capture the region within which the fluid must react to the central solid particle to present an unperturbed flow to the neighboring cells. This extension is not an accurate depiction of concentrated suspensions (we take no account of the anisotropy caused by the local interactions with neighboring particles) but it does have the potential to capture the divergence of viscosity at close packing $\phi \rightarrow \phi_{m}$. As illustrated in Fig. 2, as $\phi \rightarrow \phi_{m}$ we impose $b \rightarrow 1$. In general, we simply decouple the two variables $\phi$ and $b$. The extra flexibility introduced by this decoupling will, as we will see later, allow us to create suspension models which are both simple and physically plausible.

\section{F. Solution strategy}

Since the second-order fluid model is derived by taking the slow-flow limit of any viscoelastic fluid, it is reasonable to consider that the Weissenberg number Wi should be small. Thus, we can 


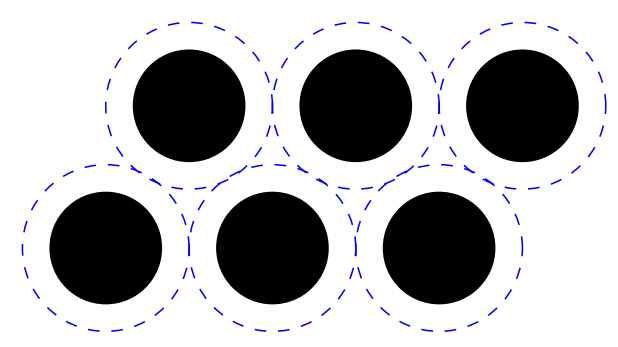

FIG. 2. Using the cell model for dense suspensions. There may be some fluid which is not contained within any cell; the radius $b$ of the sphere of influence now becomes a semiempirical measure of how close neighboring cells may be.

expand all variables $Q$ as perturbation series about the Newtonian case $\mathrm{Wi}=0$ :

$$
Q=Q^{0}+\mathrm{Wi} Q^{1}+O\left(\mathrm{Wi}^{2}\right) .
$$

We solve at each order in turn; and as one might expect, the leading-order system is precisely that of a suspension in a Newtonian fluid. We discuss the Newtonian case in Sec. III and the full second-order fluid in Sec. IV.

The governing equations become, at leading order,

$$
\begin{gathered}
\nabla \cdot \mathbf{v}^{0}=0, \\
-\nabla p^{0}+\nabla^{2} \mathbf{v}^{0}=0,
\end{gathered}
$$

with boundary conditions

$$
\begin{array}{lll}
\mathbf{v}^{0}=\mathbf{0} & \text { on } & r=1 \\
\mathbf{v}^{0}=\mathbf{u}^{\infty} & \text { on } & r=b
\end{array}
$$

and at order $\mathrm{Wi}$,

$$
\begin{gathered}
\nabla \cdot \mathbf{v}^{1}=0, \\
-\nabla p^{1}+\nabla^{2} \mathbf{v}^{1}=-\nabla \cdot\left(\epsilon \mathbf{A}^{0}+4 \mathbf{E}^{0} \cdot \mathbf{E}^{0}\right),
\end{gathered}
$$

in which $\mathbf{E}^{0}$ and $\mathbf{A}^{0}$ are defined in terms of $\mathbf{v}^{0}$ as in Eqs. (8) and (9), with boundary conditions

$$
\begin{array}{lll}
\mathbf{v}^{1}=\mathbf{0} & \text { on } & r=1, \\
\mathbf{v}^{1}=\mathbf{0} & \text { on } & r=b .
\end{array}
$$

\section{NEWTONIAN MATRIX}

In this section we will describe our method for calculating local velocity, pressure, and stress fields as a function of the boundary forcing data $\mathbf{E}^{\infty}$ and $\boldsymbol{\Omega}^{\infty}$. We will deduce macroscopic viscosity values, reproducing results of Ref. [41], and then show how we can use real suspension data to motivate an empirical relation between $b$ and $\phi$.

\section{A. Solving the flow problem}

Because of the linearity of the Stokes equations, the local velocity and pressure fields can only depend linearly on the boundary forcing: Thus, generically,

$$
\begin{gathered}
v_{i}(\mathbf{x})=\alpha_{i j k}^{(1)}(\mathbf{x}) E_{j k}^{\infty}+\alpha_{i j k}^{(2)}(\mathbf{x}) \Omega_{j k}^{\infty}, \\
p(\mathbf{x})=\beta_{j k}^{(1)}(\mathbf{x}) E_{j k}^{\infty}+\beta_{j k}^{(2)}(\mathbf{x}) \Omega_{j k}^{\infty} .
\end{gathered}
$$


Because of the spherical symmetry of the problem, the only distinguished vector direction is $\mathbf{x}$, which means we can specify the tensors $\boldsymbol{\alpha}, \boldsymbol{\beta}$ in terms of this vector and of unknown scalar functions of $r=|\mathbf{x}|$ :

$$
\begin{gathered}
v_{i}=f(r) E_{i k}^{\infty} x_{k}+g(r) E_{j k}^{\infty} x_{i} x_{j} x_{k}+h(r) \Omega_{i k}^{\infty} x_{k}, \\
p=q(r) E_{j k}^{\infty} x_{j} x_{k} .
\end{gathered}
$$

The inclusion of the rotation term $\boldsymbol{\Omega}$ here is different from the standard method used in the literature. For a Newtonian fluid this term could simply be eliminated by changing the frame of reference to one rotating with the flow, but when we move on to the second-order fluid in Sec. IV this term will become more important.

The velocity boundary conditions from Eqs. (16) and (17) become the scalar set:

$$
f(1)=0, \quad f(b)=1 ; \quad g(1)=0, \quad g(b)=0 ; \quad h(1)=1, \quad h(b)=1 .
$$

Substituting our new velocity forms into the mass conservation Eq. (5), we obtain the first-order differential equation

$$
\frac{f^{\prime}(r)}{r}+r g^{\prime}(r)+5 g(r)=0 .
$$

We take the curl of the momentum Eq. (12) to eliminate the pressure, forming the vorticity equation. Substitution of the velocity Eq. (36) then yields two equations (from the coefficients of the independent expressions depending on $\mathbf{E}$ and $\boldsymbol{\Omega})$ :

$$
\frac{f^{\prime \prime \prime}(r)}{r}+\frac{4 f^{\prime \prime}(r)}{r^{2}}-\frac{4 f^{\prime}(r)}{r^{3}}-2 g^{\prime \prime}(r)-\frac{12 g^{\prime}(r)}{r}=0, \quad h^{\prime \prime}(r)+\frac{4 h^{\prime}(r)}{r}=0 .
$$

Once these differential equations have been solved for $f(r), g(r)$, and $h(r)$ we can reconstruct the pressure function $q(r)$ using the original momentum equation:

$$
q(r)=\frac{\eta_{0}}{2}\left[f^{\prime \prime}(r)+\frac{4 f^{\prime}(r)}{r}+4 g(r)\right] .
$$

Solving, we find the radial functions

$$
\begin{aligned}
& f(r)=-\frac{5 c_{1} r^{2}}{2}-\frac{2 c_{3}}{5 r^{5}}+c_{4}, \\
& g(r)=c_{1}+\frac{c_{2}}{r^{5}}+\frac{c_{3}}{r^{7}}, \\
& h(r)=1, \\
& q(r)=\eta_{0}\left(-\frac{21 c_{1}}{2}+\frac{2 c_{2}}{r^{5}}\right),
\end{aligned}
$$

in which the constants $c_{i}$ are defined in terms of $b$ :

$$
\begin{aligned}
& c_{1}=\frac{10 b^{3}(1+b)}{(b-1)^{3}\left(4+16 b+40 b^{2}+55 b^{3}+40 b^{4}+16 b^{5}+4 b^{6}\right)}, \\
& c_{2}=-\frac{10 b^{3}\left(1+b+b^{2}+b^{3}+b^{4}+b^{5}+b^{6}\right)}{(b-1)^{3}\left(4+16 b+40 b^{2}+55 b^{3}+40 b^{4}+16 b^{5}+4 b^{6}\right)}, \\
& c_{3}=\frac{10 b^{5}\left(1+b+b^{2}+b^{3}+b^{4}\right)}{(b-1)^{3}\left(4+16 b+40 b^{2}+55 b^{3}+40 b^{4}+16 b^{5}+4 b^{6}\right)}, \\
& c_{4}=\frac{b^{3}\left(25+25 b+4 b^{2}+4 b^{3}+4 b^{4}+4 b^{5}+4 b^{6}\right)}{(b-1)^{3}\left(4+16 b+40 b^{2}+55 b^{3}+40 b^{4}+16 b^{5}+4 b^{6}\right)} .
\end{aligned}
$$

These and any further computations were calculated using the Mathematica software. 


\section{B. Bulk stress}

As discussed in Sec. II D, we can write the average deviatoric stress in the system as

$$
\langle\widehat{\boldsymbol{\sigma}}\rangle=2\langle\mathbf{E}\rangle+\frac{3 \phi}{4 \pi} \widehat{\mathbf{S}},
$$

[which is Eq. (20) in the limit Wi $\rightarrow$ 0], along with the stresslet definition from Eq. (21):

$$
S_{i j}=\frac{1}{2} \int_{r=1}\left(\sigma_{i k} \hat{n}_{j}+\sigma_{k j} \hat{n}_{i}\right) \hat{n}_{k} d S
$$

Using the velocity functions Eq. (42) and their derived stresses, we obtain

$$
\left\langle\widehat{\sigma_{i j}}\right\rangle=2\left[1+\frac{10 b^{3}\left(1+b+b^{2}+b^{3}+b^{4}+b^{5}+b^{6}\right) \phi}{(b-1)^{3}\left(4+16 b+40 b^{2}+55 b^{3}+40 b^{4}+16 b^{5}+4 b^{6}\right)}\right] E_{i j}^{\infty} .
$$

In dimensional terms, then

$$
\eta_{\mathrm{eff}}=\eta_{0}\left[1+\frac{10 b^{3}\left(1+b+b^{2}+b^{3}+b^{4}+b^{5}+b^{6}\right) \phi}{(b-1)^{3}\left(4+16 b+40 b^{2}+55 b^{3}+40 b^{4}+16 b^{5}+4 b^{6}\right)}\right] .
$$

In the limit $b \rightarrow \infty$, this reduces correctly to Einstein's viscosity $\eta_{\mathrm{eff}}=1+5 \phi / 2$; it also matches Eq. (30) of Sherwood [41] if we make the additional assumption that $\phi=b^{-3}$.

However, in this work we have deliberately not assumed a relation between $b$ and $\phi$. The reason is simple: We wish to use this freedom to make our predictions more physically plausible.

For a monodisperse suspension of spherical particles, it is well known that the KriegerDougherty relation Eq. (3)

$$
\eta_{\text {eff }}=\eta_{0}\left(1-\frac{\phi}{\phi_{m}}\right)^{-5 \phi_{m} / 2}
$$

gives viscosity predictions which are a good fit to experimental data [22]. We can choose the function $b(\phi)$ so that our cell model's effective viscosity precisely matches Krieger-Dougherty:

$$
\frac{10 b^{3}\left(1+b+b^{2}+b^{3}+b^{4}+b^{5}+b^{6}\right) \phi}{(b-1)^{3}\left(4+16 b+40 b^{2}+55 b^{3}+40 b^{4}+16 b^{5}+4 b^{6}\right)}=\left(1-\frac{\phi}{\phi_{m}}\right)^{-5 \phi_{m} / 2}-1 .
$$

This implicit expression for $b$ cannot be solved analytically, but we graph the resulting function in Fig. 3. Its asymptotic behavior is

$$
\begin{gathered}
b(\phi) \sim \frac{5}{2}\left(\frac{25}{8}+\frac{5}{4 \phi_{m}}\right)^{-1 / 3} \phi^{-1 / 3} \text { as } \phi \rightarrow 0, \\
b(\phi) \sim 1+\left(\frac{2 \phi_{m}{ }^{1-5 \phi_{m} / 2}}{5}\right)^{1 / 3}\left(\phi_{m}-\phi\right)^{5 \phi_{m} / 6} \text { as } \phi \rightarrow \phi_{m},
\end{gathered}
$$

and in Table I we tabulate its value at intermediate concentrations. The ratio between $b(\phi)$ and $\phi^{-1 / 3}$, the assumed value for $b$ in the dilute limit is also plotted in Fig. 4. We also tabulate the values of $\phi^{1 / 3} b(\phi)$ at intermediate concentrations in Table II.

This empirical function $b(\phi)$ modifies the volume of fluid available to each particle within the mean-field approximation. As $\phi \rightarrow \phi_{m}$, in a real suspension system, the gap between each particle and its nearest neighbors decreases to zero: this fit function captures that behavior by reducing the volume of the cellular fluid phase to zero. We can think of this, qualitatively, as using a proxy for many-body interactions, in a way that still fits within the mean-field framework. It then makes sense to use the Krieger-Dougherty viscosity to choose $b(\phi)$ so that our resulting constitutive model best fits experimental data. 


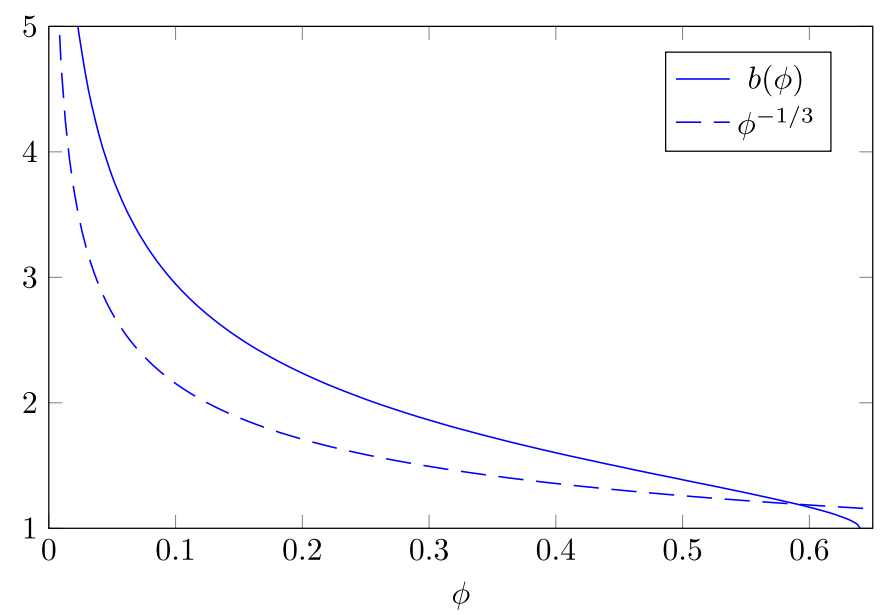

FIG. 3. Solid line: The empirical function $b(\phi)$ defining the cell radius as a function of the solid volume fraction $\phi$ to mimic the Krieger-Dougherty viscosity. We have taken $\phi_{m}$ as 0.64 , the maximum random packing fraction for monodisperse spheres. The dotted line shows the standard assumption $b(\phi)=\phi^{-1 / 3}$.

We provide a plot in Fig. 5 which shows effective viscosity obtained using numerical analysis [43], dilute theory [30], and the cell model approach for semidilute values of solid volume fraction. The numerical data points were obtained by calculating flow quantities about one particle in the center of a cubic domain, with an Oldroyd-B background fluid. We observe that both theoretical approaches have a good comparison with the data points at $\phi=0.05$, although the KriegerDougherty equation is slightly closer at all Wi. However, as we reach the higher value of solid volume fraction $\phi=0.1$, our cell model approach combined with Krieger-Dougherty matching clearly fits the numerical data better. We think that this improvement in reproducing numerical data, having started from dilute assumptions in our analysis, is encouraging and may provide a means to predict other viscometric functions at larger concentrations than $\phi \ll 1$.

In the next section we will extend our analysis to a second-order suspending fluid. With the change of suspending fluid comes a question as to whether close-pair interactions are comparably important in Newtonian and second-order fluids. There is some evidence that they may not be-for example, viscoelastic fluids often show particle chaining, which means they may have more close pairs than corresponding Newtonian fluids, and there is some far-field theoretical work [44] predicting attractive interactions in a second-order fluid. However, given that the second-order fluid

TABLE I. Numerical values for the empirical relation $b(\phi)$ defined in Eq. (49), taking $\phi_{m}=0.64$.

\begin{tabular}{|c|c|c|c|c|c|c|c|c|c|c|c|c|c|}
\hline$\phi$ & $b(\phi)$ & $\phi$ & $b(\phi)$ & $\phi$ & $b(\phi)$ & $\phi$ & $b(\phi)$ & $\phi$ & $b(\phi)$ & $\phi$ & $b(\phi)$ & $\phi$ & $b(\phi)$ \\
\hline 0.00 & $\infty$ & 0.10 & 2.947 & 0.20 & 2.236 & 0.30 & 1.863 & 0.40 & 1.602 & 0.50 & 1.387 & 0.60 & 1.168 \\
\hline 0.01 & 6.669 & 0.11 & 2.842 & 0.21 & 2.190 & 0.31 & 1.833 & 0.41 & 1.579 & 0.51 & 1.366 & 0.61 & 1.141 \\
\hline 0.02 & 5.255 & 0.12 & 2.748 & 0.22 & 2.146 & 0.32 & 1.804 & 0.42 & 1.557 & 0.52 & 1.346 & 0.62 & 1.111 \\
\hline 0.03 & 4.562 & 0.13 & 2.664 & 0.23 & 2.105 & 0.33 & 1.777 & 0.43 & 1.535 & 0.53 & 1.325 & 0.63 & 1.074 \\
\hline 0.04 & 4.121 & 0.14 & 2.587 & 0.24 & 2.066 & 0.34 & 1.750 & 0.44 & 1.513 & 0.54 & 1.304 & 0.64 & 1.000 \\
\hline 0.05 & 3.805 & 0.15 & 2.517 & 0.25 & 2.028 & 0.35 & 1.724 & 0.45 & 1.492 & 0.55 & 1.283 & & \\
\hline 0.06 & 3.562 & 0.16 & 2.452 & 0.26 & 1.992 & 0.36 & 1.698 & 0.46 & 1.470 & 0.56 & 1.261 & & \\
\hline 0.07 & 3.367 & 0.17 & 2.393 & 0.27 & 1.958 & 0.37 & 1.673 & 0.47 & 1.449 & 0.57 & 1.239 & & \\
\hline 0.08 & 3.205 & 0.18 & 2.337 & 0.28 & 1.925 & 0.38 & 1.649 & 0.48 & 1.428 & 0.58 & 1.216 & & \\
\hline 0.09 & 3.067 & 0.19 & 2.285 & 0.29 & 1.893 & 0.39 & 1.625 & 0.49 & 1.408 & 0.59 & 1.193 & & \\
\hline
\end{tabular}




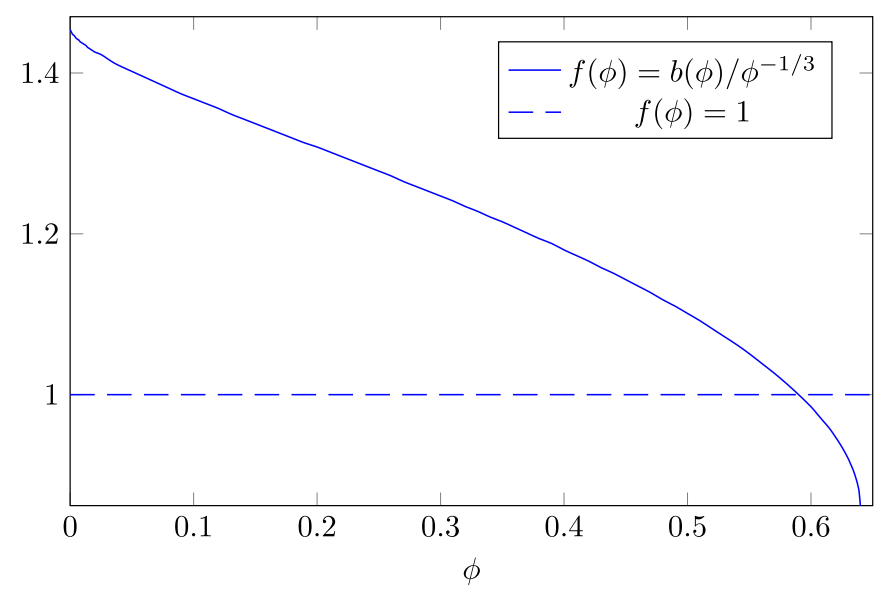

FIG. 4. Solid line: The function $f(\phi)$ is defined as the ratio between $b(\phi)$ and $\phi^{-1 / 3}$. The empirical function $b(\phi)$ defines the cell radius as a function of the solid volume fraction $\phi$ to mimic the Krieger-Dougherty viscosity. We have taken $\phi_{m}$ as 0.64 , the maximum random packing fraction for monodisperse spheres. The dotted line shows the same function $f(\phi)$ under the standard assumption $b(\phi)=\phi^{-1 / 3}$.

is a first viscoelastic adjustment to the Newtonian model, it seems natural to use the effective volumes yielded by Krieger-Dougherty as a starting point.

\section{SECOND-ORDER FLUID MATRIX}

In this section, we calculate the local velocity and pressure fields at $O(\mathrm{Wi})$. They serve to find the perturbation to the stresslet as defined in Eq. (21), which in turn contributes to the additional bulk stress. We will calculate the macroscopic stress at this order of our perturbation series, and deduce values of both material parameters in bulk. We then use our empirical $b(\phi)$ function to produce concentration dependent normal stress differences, and show their behavior in a simple shear flow.

\section{A. Solving the flow problem at $O(\mathrm{Wi})$}

If we refer back to Sec. II F, we see that Eq. (32) at order Wi is the Stokes equation with a forcing term which is quadratic in the boundary data $\mathbf{E}^{\infty}$ and $\boldsymbol{\Omega}^{\infty}$. Any pressure or velocity term at this order

TABLE II. Numerical values for the ratio $\phi^{1 / 3} b(\phi)$ using the empirical relation $b(\phi)$ defined in Eq. (49), taking $\phi_{m}=0.64$.

\begin{tabular}{lccccccccccccc}
\hline \hline$\phi$ & $\phi^{1 / 3} b$ & $\phi$ & $\phi^{1 / 3} b$ & $\phi$ & $\phi^{1 / 3} b$ & $\phi$ & $\phi^{1 / 3} b$ & $\phi$ & $\phi^{1 / 3} b$ & $\phi$ & $\phi^{1 / 3} b$ & $\phi$ & $\phi^{1 / 3} b$ \\
\hline 0.00 & 1.454 & 0.10 & 1.368 & 0.20 & 1.308 & 0.30 & 1.247 & 0.40 & 1.180 & 0.50 & 1.101 & 0.60 & 0.985 \\
0.01 & 1.437 & 0.11 & 1.362 & 0.21 & 1.302 & 0.31 & 1.241 & 0.41 & 1.173 & 0.51 & 1.092 & 0.61 & 0.967 \\
0.02 & 1.426 & 0.12 & 1.356 & 0.22 & 1.296 & 0.32 & 1.234 & 0.42 & 1.166 & 0.52 & 1.082 & 0.62 & 0.947 \\
0.03 & 1.418 & 0.13 & 1.349 & 0.23 & 1.290 & 0.33 & 1.228 & 0.43 & 1.158 & 0.53 & 1.072 & 0.63 & 0.921 \\
0.04 & 1.409 & 0.14 & 1.343 & 0.24 & 1.284 & 0.34 & 1.221 & 0.44 & 1.151 & 0.54 & 1.062 & 0.64 & 0.862 \\
0.05 & 1.402 & 0.15 & 1.337 & 0.25 & 1.278 & 0.35 & 1.215 & 0.45 & 1.143 & 0.55 & 1.051 & & \\
0.06 & 1.395 & 0.16 & 1.331 & 0.26 & 1.272 & 0.36 & 1.208 & 0.46 & 1.135 & 0.56 & 1.039 & & \\
0.07 & 1.388 & 0.17 & 1.325 & 0.27 & 1.265 & 0.37 & 1.201 & 0.47 & 1.127 & 0.57 & 1.027 & & \\
0.08 & 1.381 & 0.18 & 1.319 & 0.28 & 1.259 & 0.38 & 1.194 & 0.48 & 1.118 & 0.58 & 1.014 & & \\
0.09 & 1.374 & 0.19 & 1.313 & 0.29 & 1.253 & 0.39 & 1.188 & 0.49 & 1.110 & 0.59 & 1.000 & & \\
\hline \hline
\end{tabular}




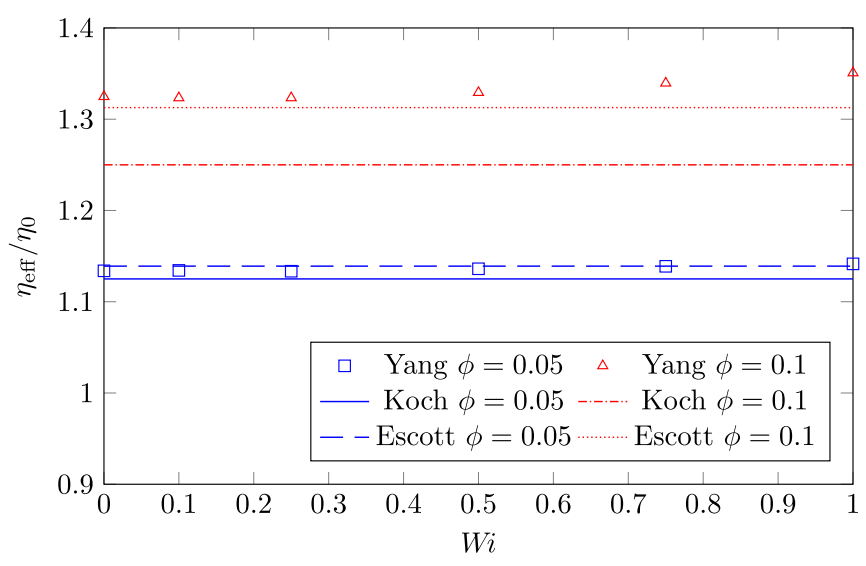

FIG. 5. A comparison of normalized effective viscosity $\eta_{\text {eff }} / \eta_{0}$ in suspension between the numerical results of Yang and Shaqfeh [43], the dilute theory [30] and our own results using $b(\phi)$. The numerical results are given by the square and triangular data points, dilute theory by the solid and long dashed lines and our cell model approach by the short dashed lines. The matching process which results in the empirical function $b(\phi)$ guarantees that $\eta_{\text {eff }}$ is given precisely by the Krieger-Dougherty Eq. (3). We show results at semidilute values of solid volume fraction $\phi=0.05$ and 0.1 .

must therefore also have a quadratic dependence on the boundary data, otherwise it will be a trivial solution. Thus, generally,

$$
\begin{aligned}
& v_{i}^{1}(\mathbf{x})=\alpha_{i j k l m}^{(1)}(\mathbf{x}) E_{j k}^{\infty} E_{l m}^{\infty}+\alpha_{i j k l m}^{(2)}(\mathbf{x}) E_{j k}^{\infty} \Omega_{l m}^{\infty}, \\
& p^{1}(\mathbf{x})=\beta_{j k l m}^{(1)}(\mathbf{x}) E_{j k}^{\infty} E_{l m}^{\infty}+\beta_{j k l m}^{(2)}(\mathbf{x}) E_{j k}^{\infty} \Omega_{l m}^{\infty} .
\end{aligned}
$$

Note that there are no $\boldsymbol{\Omega} \boldsymbol{\Omega}$ terms generated by the forcing term in Eq. (32), and therefore any dependence on this tensor combination in $v_{i}^{1}(\mathbf{x})$ or $p^{1}(\mathbf{x})$ would be homogeneous.

As aforementioned in Sec. III A, the spherical symmetry of the problem allows for only one choice of vector direction $\mathbf{x}$. Therefore, we can specify the tensors $\boldsymbol{\alpha}, \boldsymbol{\beta}$ in terms of this vector and unknown scalar functions of $r=|\mathbf{x}|$ :

$$
\begin{aligned}
v_{i}^{1}= & V_{1}(r) E_{j k}^{\infty} E_{l m}^{\infty} x_{i} x_{j} x_{k} x_{l} x_{m}+V_{2}(r) E_{j k}^{\infty} E_{k l}^{\infty} x_{i} x_{j} x_{l}+V_{3}(r) E_{j k}^{\infty} E_{j k}^{\infty} x_{i}+V_{4}(r) E_{j k}^{\infty} E_{i l}^{\infty} x_{j} x_{k} x_{l} \\
& +V_{5}(r) E_{i k}^{\infty} E_{k l}^{\infty} x_{l}+V_{6}(r) E_{j k}^{\infty} \Omega_{k l}^{\infty} x_{i} x_{j} x_{k}+V_{7}(r) E_{i k}^{\infty} \Omega_{k l}^{\infty} x_{l}+V_{8}(r) \Omega_{i k}^{\infty} E_{k l}^{\infty} x_{l}, \\
p^{1}= & P_{1}(r) E_{j k}^{\infty} E_{l m}^{\infty} x_{j} x_{k} x_{l} x_{m}+P_{2}(r) E_{j k}^{\infty} E_{k l}^{\infty} x_{j} x_{l}+P_{3}(r) E_{j k}^{\infty} E_{j k}^{\infty}+P_{4}(r) E_{j k}^{\infty} \Omega_{k l}^{\infty} x_{j} x_{l} .
\end{aligned}
$$

The governing equations which these scalar functions must satisfy are unwieldy, and are provided in Appendix A. Their solution yields the radial functions

$$
\begin{aligned}
V_{1}(r)= & -\frac{C_{1}}{11 r^{11}}+C_{2}-\frac{C_{10}}{18 r^{9}}+\frac{11(1+\epsilon) c_{1} c_{2}}{6 r^{5}}+\frac{7(1+\epsilon) c_{1} c_{3}}{2 r^{7}}+\frac{8(1+\epsilon) c_{2}^{2}}{r^{10}} \\
& -\frac{4(1+\epsilon) c_{2} c_{3}}{r^{12}}+\frac{7(2+3 \epsilon) c_{3} c_{4}}{r^{9}}, \\
V_{2}(r)= & \frac{4 C_{1}}{99 r^{9}}-\frac{4\left(7 C_{2}+C_{11}\right) r^{2}}{63}-\frac{C_{3}}{7 r^{7}}+C_{4}-\frac{C_{12}}{10 r^{5}}+\frac{(53+27 \epsilon) c_{1}^{2}}{21}+\frac{17(1+\epsilon) c_{1} c_{2}}{9 r^{3}} \\
& +\frac{(162+215 \epsilon) c_{1} c_{3}-10(3+5 \epsilon) c_{2} c_{4}}{5 r^{5}}-\frac{6(1+\epsilon) c_{2}^{2}}{r^{8}}+\frac{4(1+\epsilon) c_{2} c_{3}}{r^{10}},
\end{aligned}
$$




$$
\begin{aligned}
& V_{3}(r)=-\frac{2 C_{1}}{693 r^{7}}+\frac{2\left(5 C_{2}+2 C_{11}\right) r^{4}}{315}+\frac{9 C_{3}+C_{10}}{315 r^{5}}-\frac{\left(3 C_{4}+C_{13}\right) r^{2}}{15}-\frac{C_{5}}{3 r^{3}}+C_{6} \\
& +\frac{(83+135 \epsilon) c_{1}^{2} r^{4}}{210}-\frac{28(1+\epsilon) c_{1} c_{2}}{9 r}-\frac{(11+20 \epsilon) c_{1} c_{4} r^{2}}{5}+\frac{2(1+\epsilon) c_{2}^{2}}{3 r^{6}} \\
& -\frac{2(2+3 \epsilon) c_{3} c_{4}}{5 r^{5}} \\
& V_{4}(r)=\frac{4 C_{1}}{99 r^{9}}-\frac{2\left(2 C_{2}-C_{11}\right) r^{2}}{9}-\frac{C_{7}}{7 r^{7}}+C_{8}-\frac{(11-15 \epsilon) c_{1}^{2} r^{2}}{6}+\frac{11(1+\epsilon) c_{1} c_{2}}{9 r^{3}} \\
& -\frac{3(1+\epsilon) c_{2}^{2}}{r^{8}}+\frac{2(1+\epsilon) c_{2} c_{3}}{r^{10}}, \\
& V_{5}(r)=-\frac{8 C_{1}}{693 r^{7}}+\frac{4\left(2 C_{2}-C_{11}\right) r^{4}}{63}+\frac{2\left(9 C_{3}+9 C_{7}+2 C_{10}\right)}{315 r^{5}}-\frac{\left(2 C_{4}+2 C_{8}-C_{13}\right) r^{2}}{5} \\
& +C_{9}-\frac{(125+177 \epsilon) c_{1}^{2} r^{4}}{42}+\frac{56(1+\epsilon) c_{1} c_{2}}{9 r}+\frac{3(11+20 \epsilon) c_{1} c_{4} r^{2}}{5} \\
& +\frac{2(1+\epsilon) c_{2}^{2}}{r^{6}}-\frac{8(2+3 \epsilon) c_{3} c_{4}}{5 r^{5}} \\
& V_{6}(r)=0, \quad V_{7}(r)=0, \quad V_{8}(r)=0, \\
& P_{1}(r)=-\frac{C_{10}}{9 r^{9}}+C_{11}+\frac{(79+120 \epsilon) c_{1} c_{2}}{r^{5}}+\frac{2(59+78 \epsilon) c_{1} c_{3}-20(1+2 \epsilon) c_{2} c_{4}}{r^{7}}+\frac{2(6+5 \epsilon) c_{2}^{2}}{r^{10}} \\
& +\frac{2(8+11 \epsilon) c_{2} c_{3}}{r^{12}}+\frac{18(2+3 \epsilon) c_{3}^{2}}{5 r^{14}} \\
& P_{2}(r)=\frac{4 C_{10}}{63 r^{7}}-\frac{4 C_{11} r^{2}}{7}-\frac{C_{12}}{5 r^{5}}+C_{13}+\frac{6(72+143 \epsilon) c_{1}^{2} r^{2}}{7}-\frac{2(23+36 \epsilon) c_{1} c_{2}}{r^{3}}+\frac{2(1+2 \epsilon) c_{2}^{2}}{r^{8}} \\
& +\frac{8(8+11 \epsilon) c_{2} c_{3}}{5 r^{10}}+\frac{24(2+3 \epsilon) c_{3}^{2}}{5 r^{12}} \\
& P_{3}(r)=-\frac{2 C_{10}}{315 r^{5}}+\frac{2 C_{11} r^{4}}{35}+\frac{C_{12}}{15 r^{3}}-\frac{C_{13} r^{2}}{3}+\frac{(1089+2120 \epsilon) c_{1}^{2} r^{4}}{140} \\
& -\frac{4(59+78 \epsilon) c_{1} c_{3}-40(1+2 \epsilon) c_{2} c_{4}}{15 r^{3}}-14(1+2 \epsilon) c_{1} c_{4} r^{2}+\frac{4(2+3 \epsilon) c_{3}^{2}}{25 r^{10}}, \\
& P_{4}(r)=-21 \epsilon c_{1}+\frac{4 \epsilon c_{2}}{r^{5}}
\end{aligned}
$$

with two sets of constants: $c_{i}$ given in Eq. (43) and $C_{i}$, new at this order, which are provided in Appendix B.

In the case of one spherical particle in an infinite volume of second-order background fluid, the solution for pressure and velocity functions is given by Peery [45]. We find that our solutions Eq. (55) match Ref. [45] under the dilute limit $b \rightarrow \infty$, less a constant pressure term $E_{j k}^{\infty} E_{j k}^{\infty}$. Since there are no boundary conditions on the pressure and only its gradient plays a role in the governing momentum equations, this constant pressure term difference between our solutions is of no consequence.

As discussed in Sec. II A, Einarsson et al. [39] provided an analysis on the so-called "hot spots," which are regions about the surface of a particle within which the straining part of a flow dominates. The suspending fluid in their case was Oldroyd-B, and the flow profile was simple shear. To demonstrate these hot spots, they provided a plot of the velocity gradient using an eigenvalue technique [40], which as well as locating these areas with a strong straining component, also 
demonstrates their close proximity to the particle. We have performed a similar analysis on our velocity gradient tensor to $O(\mathrm{Wi})$, and found a very similar profile: strong areas of strain fore and aft the particle and rotation dominated areas above and below. This qualitative agreement shows that, for at least the dilute case, the general profile of analytical results found in an infinite fluid can be equally captured using a bounded spherical domain.

\section{B. $O(\mathrm{Wi})$ correction to bulk stress}

We saw in Sec. II D that up to $O(\mathrm{Wi})$, the symmetric deviatoric stress in the system can be written as

$$
\langle\widehat{\boldsymbol{\sigma}}\rangle=2\langle\mathbf{E}\rangle+\operatorname{Wi}(\epsilon\langle\widehat{\mathbf{A}}\rangle+4\langle\widehat{\mathbf{E} \cdot \mathbf{E}}\rangle)+\frac{3 \phi}{4 \pi} \widehat{\mathbf{S}}
$$

from Eq. (20), with the definitions of second-order Rivlin-Ericksen tensor $\mathbf{A}$ and stresslet $\mathbf{S}$ given in Eqs. (23) and (21), respectively. While the mean polymer stress term here depends only on the Newtonian flow, we must take the $O(\mathrm{Wi})$ contribution to the local pressure and velocity fields to find the stresslet term. Therefore, using the solutions for velocity Eqs. (42) and (55) in the Newtonian and second-order matrices, respectively, we are able to calculate the average stress in the form

$$
\left\langle\widehat{\sigma_{i j}}\right\rangle=2 \frac{\eta_{\mathrm{eff}}}{\eta_{0}} E_{i j}^{\infty}+\mathrm{Wi}\left(\Sigma_{0} \widehat{E_{i k}^{\infty} \Omega_{k j}^{\infty}}+\Sigma_{1} \widehat{E_{i k}^{\infty} E_{k j}^{\infty}}\right) .
$$

The effective viscosity $\eta_{\text {eff }}$ is that given in Eq. (47). The new constitutive functions $\Sigma_{0}$ and $\Sigma_{1}$ are provided explicitly in Appendix C. They depend on the second-order fluid parameters $\alpha_{0}$ and $\alpha_{1}$ and, of course, on the cell radius $b$.

While we have used the $O(\mathrm{Wi})$ velocity and pressure terms to calculate the functions $\Sigma_{0}$ and $\Sigma_{1}$, one may use the generalized reciprocal theorem as demonstrated by Koch and Subramanian [30]. The benefit of this method is that we can calculate the $O(\mathrm{Wi})$ stresslet $\widehat{\mathbf{S}}^{1}$ without the need to find $v_{i}^{1}$ and $p^{1}$, which can be time consuming and unnecessary if the bulk stress terms are the only required quantities. We have performed both techniques in the volume contained by our cell model to derive bulk stress coefficients and verify that both methods give the same result.

In Sec. III B we found the effective viscosity of our suspension in a Newtonian background fluid, which is the one material function. At second order, we have introduced two more parameters $\alpha_{0}$ and $\alpha_{1}$, which in bulk correspond to two further material functions

$$
\alpha_{0, \mathrm{eff}}=\frac{\Sigma_{0}}{4 \epsilon} \alpha_{0}, \quad \alpha_{1, \mathrm{eff}}=\frac{\Sigma_{1}-\Sigma_{0}}{4} \alpha_{1}
$$

given in dimensional terms as functions of $\Sigma_{0}, \Sigma_{1}$ and the initial constants $\alpha_{0}, \alpha_{1}$.

Explicitly then, we obtain

$$
\begin{gathered}
\alpha_{0, \mathrm{eff}}=\left[1+\frac{10 b^{3}\left(1+b+b^{2}+b^{3}+b^{4}+b^{5}+b^{6}\right) \phi}{(b-1)^{3}\left(4+16 b+40 b^{2}+55 b^{3}+40 b^{4}+16 b^{5}+4 b^{6}\right)}\right] \alpha_{0}, \\
\alpha_{1, \mathrm{eff}}=\left[1+\frac{\Lambda_{\phi} \phi}{7(b-1)^{4}\left(4+16 b+40 b^{2}+55 b^{3}+40 b^{4}+16 b^{5}+4 b^{6}\right)^{3}}\right] \alpha_{1},
\end{gathered}
$$

in which we have introduced

$$
\begin{aligned}
\Lambda_{\phi}= & 15 b^{3}\left(-32-256 b-1152 b^{2}-3140 b^{3}-4960 b^{4}-3336 b^{5}+4802 b^{6}+18964 b^{7}\right. \\
& +34140 b^{8}+45760 b^{9}+53768 b^{10}+59004 b^{11}+59508 b^{12}+52185 b^{13}+37320 b^{14} \\
& \left.+20800 b^{15}+8900 b^{16}+2880 b^{17}+640 b^{18}+80 b^{19}\right)+10 b^{3}\left(88+704 b+3168 b^{2}\right. \\
& +10060 b^{3}+25040 b^{4}+49344 b^{5}+78442 b^{6}+104852 b^{7}+122604 b^{8}+128848 b^{9} \\
& +123128 b^{10}+104844 b^{11}+75892 b^{12}+44597 b^{13}+20304 b^{14}+6640 b^{15}+1460 b^{16} \\
& \left.+288 b^{17}+64 b^{18}+8 b^{19}\right) \epsilon .
\end{aligned}
$$


We note here that $\alpha_{0, \text { eff }} / \alpha_{0}=\eta_{\text {eff }} / \eta_{0}$, which can be seen in Eqs. (47) and (59).

In the limit $b \rightarrow \infty$, these functions reduce to

$$
\alpha_{0, \mathrm{eff}}=\left(1+\frac{5 \phi}{2}\right) \alpha_{0}, \quad \alpha_{1, \mathrm{eff}}=\left[1+\frac{5(15+\epsilon) \phi}{28}\right] \alpha_{1},
$$

and our constitutive stress functions behave as

$$
\Sigma_{0}=4 \epsilon+10 \epsilon \phi, \quad \Sigma_{1}=4(1+\epsilon)+\frac{75(1+\epsilon) \phi}{7},
$$

which matches Eqs. (68) and (69) of Ref. [30].

\section{Model for a macroscopic suspension}

As we saw in Sec. II B, the governing equation for a second-order fluid is

$$
\boldsymbol{\sigma}=-p \mathbf{I}+2 \eta_{0} \mathbf{E}+\alpha_{0} \mathbf{A}+4 \alpha_{1} \mathbf{E} \cdot \mathbf{E},
$$

from Eq. (7). Using this equation, we have calculated the local stress about a single particle, and further calculated the bulk value for stress over the whole suspension. In addition, we found that in bulk, the material parameters $\eta_{0}, \alpha_{0}$ and $\alpha_{1}$ take on effective values $\eta_{\text {eff }}, \alpha_{0, \text { eff }}$, and $\alpha_{1 \text {,eff }}$, respectively, all of which represent the parameters for our suspension at the macroscopic level. Thus, we find that a suspension of spherical particles in second-order fluid can be described fully by its dimensional stress equation,

$$
\boldsymbol{\sigma}=-p^{\infty} \mathbf{I}+2 \eta_{\mathrm{eff}} \mathbf{E}^{\infty}+\alpha_{0, \mathrm{eff}} \mathbf{A}^{\infty}+4 \alpha_{1, \mathrm{eff}} \mathbf{E}^{\infty} \cdot \mathbf{E}^{\infty}
$$

Hence, in nondimensional terms the symmetric deviatoric stress is

$$
\widehat{\sigma_{i j}}=2 \frac{\eta_{\mathrm{eff}}}{\eta_{0}} E_{i j}^{\infty}+\mathrm{Wi}\left[4 \epsilon \frac{\alpha_{0, \mathrm{eff}}}{\alpha_{0}} \widehat{E_{i k}^{\infty} \Omega_{k j}^{\infty}}+4\left(\frac{\alpha_{1, \mathrm{eff}}}{\alpha_{1}}+\epsilon \frac{\alpha_{0, \mathrm{eff}}}{\alpha_{0}}\right) \widehat{E_{i k}^{\infty} E_{k j}^{\infty}}\right] .
$$

We now build on the work of Sec. III B, where we chose a function $b(\phi)$ to match our effective viscosity to the empirical Krieger-Dougherty equation. Putting this value of $b(\phi)$ into Eqs. (59) and (60) results in material functions which will be dependent solely on the parameter $\epsilon$ and the concentration $\phi$, shown in Figures 6(a) and 6(b), respectively. All results presented in the rest of this section will use this semiempirical relation of $b(\phi)$.

We noted previously that the ratio $\alpha_{0, \text { eff }} / \alpha_{0}$ is identical to $\eta_{\text {eff }} / \eta_{0}$. Therefore, as a direct consequence of our effective viscosity matching in Sec. III B, the graph in Fig. 6(a) is precisely the plot of the Krieger-Dougherty viscosity. We see a similar profile in Fig. 6 (b) for $\alpha_{1, \text { eff }} / \alpha_{1}$, except that the function value increases with decreasing absolute value of $\epsilon$.

Ho and Leal [46] state based on experimental results that for real, homogeneous fluids $\epsilon \leqslant-0.5$, so we take this as the upper limit for $\epsilon$. Further, we investigate the macroscopic suspension to reason a lower limit for $\epsilon$, which involves considering the effective parameter $\epsilon_{\text {eff }}=\alpha_{0 \text {,eff }} / \alpha_{1 \text {,eff }}$. Since both $\alpha_{0, \text { eff }}$ and $\alpha_{1, \text { eff }}$ are nonsingular at all concentrations less than jamming, we expect the same behavior for effective parameter $\epsilon_{\text {eff }}$, implying that $\alpha_{1 \text {,eff }}$, given in Eq. (60) must not change sign. This occurs at values of $\epsilon$ for which $\alpha_{1, \text { eff }} / \alpha_{1}$ diverges to $-\infty$ in the jamming limit, $b \rightarrow 1$. It can be shown analytically using Residue analysis that

$$
\frac{\alpha_{1, \mathrm{eff}}}{\alpha_{1}}=\frac{3(9+14 \epsilon) \phi}{175(b-1)^{4}}+O\left[\frac{1}{(b-1)^{3}}\right]
$$

i.e., our effective parameter $\alpha_{1 \text {,eff }}$ will change sign for $\epsilon<-9 / 14$ as we approach the maximum sphere packing limit. We therefore consider the range of $\epsilon$ to be $\epsilon \in(-9 / 14,-1 / 2)$. 


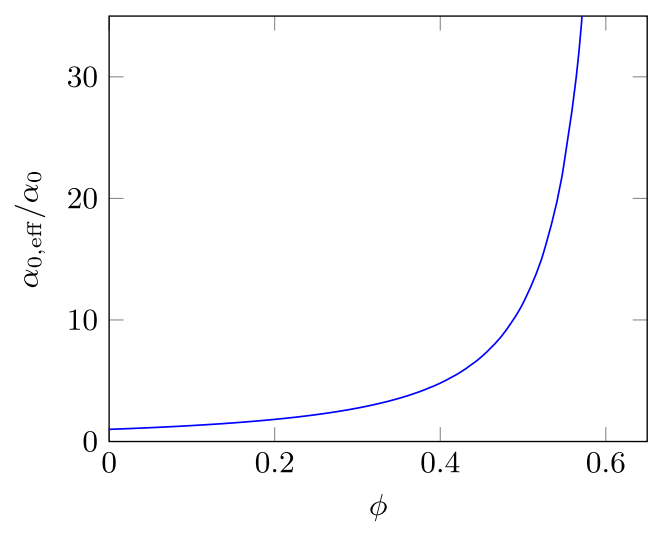

(a)

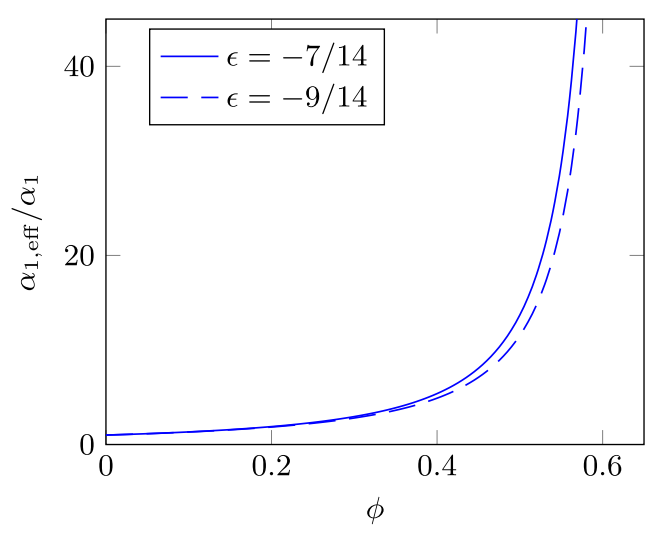

(b)

FIG. 6. The normalized effective material functions $\alpha_{0, \text { eff }} / \alpha_{0}$ and $\alpha_{1, \text { eff }} / \alpha_{1}$ as a function of solid volume fraction $\phi$ using our Krieger-Dougherty matching $b(\phi)$ shown in panels (a) and (b), respectively. We have taken $\phi_{m}$ as 0.64 , the maximum random packing fraction for monodisperse spheres. In panel (a), we see that the graph coincides exactly with the Krieger-Dougherty form (which we imposed for viscosity). We have taken two choices of parameter in panel (b) to show the divergent behavior: $\epsilon=-7 / 14$, the solid line and $\epsilon=-9 / 14$, the dashed line.

\section{Behaviour in shear flow} by

The first and second normal stress differences, denoted by $N_{1}$ and $N_{2}$, respectively, are defined

$$
N_{1}=\sigma_{x x}-\sigma_{y y}, \quad N_{2}=\sigma_{y y}-\sigma_{z z},
$$

for a unidirectional flow in direction $\hat{x}$ with shear gradient varying in $\hat{y}$. One such background flow which satisfies these definitions is

$$
\mathbf{u}^{\infty}=\dot{\gamma}(y, 0,0)
$$

with $\dot{\gamma}$ dimensionless shear rate, acting on our solid particle suspension in second-order fluid. We can calculate the macroscopic $N_{1}$ and $N_{2}$ directly from the symmetric deviatoric stress Eq. (66). Therefore, we shall simply calculate the constitutive matrices in the stress equation directly from the boundary data.

The velocity field given in Eq. (69) generates the following matrices for velocity gradient $\mathbf{L}^{\infty}$, rate of strain $\mathbf{E}^{\infty}$ and rate of rotation $\mathbf{\Omega}^{\infty}$ :

$\mathbf{L}^{\infty}=\left(\begin{array}{lll}0 & \dot{\gamma} & 0 \\ 0 & 0 & 0 \\ 0 & 0 & 0\end{array}\right), \mathbf{E}^{\infty}=\left(\begin{array}{ccc}0 & \dot{\gamma} / 2 & 0 \\ \dot{\gamma} / 2 & 0 & 0 \\ 0 & 0 & 0\end{array}\right), \boldsymbol{\Omega}^{\infty}=\left(\begin{array}{ccc}0 & \dot{\gamma} / 2 & 0 \\ -\dot{\gamma} / 2 & 0 & 0 \\ 0 & 0 & 0\end{array}\right)$.

We will also need the quadratic tensor combinations

$$
\widehat{\mathbf{E}^{\infty} \cdot \boldsymbol{\Omega}^{\infty}}=\frac{\dot{\gamma}^{2}}{4}\left(\begin{array}{ccc}
-1 & 0 & 0 \\
0 & 1 & 0 \\
0 & 0 & 0
\end{array}\right), \quad \widehat{\mathbf{E}^{\infty} \cdot \mathbf{E}^{\infty}}=\frac{\dot{\gamma}^{2}}{12}\left(\begin{array}{ccc}
1 & 0 & 0 \\
0 & 1 & 0 \\
0 & 0 & -2
\end{array}\right),
$$

which govern the $O(\mathrm{Wi})$ term.

Using the matrices above, Eq. (68) yields

$$
N_{1}=-2 \dot{\gamma}^{2} \epsilon \frac{\alpha_{0, \text { eff }}}{\alpha_{0}} \mathrm{Wi}, \quad N_{2}=\dot{\gamma}^{2}\left(\frac{\alpha_{1, \text { eff }}}{\alpha_{1}}+2 \epsilon \frac{\alpha_{0, \text { eff }}}{\alpha_{0}}\right) \mathrm{Wi},
$$


TABLE III. Table showing the dependence of all material functions on Weissenberg number, along with a direct comparison to Yang et al. [47]. The corresponding choice of parameter is $\epsilon=-8 / 14$ to compare with the numerical results, and we take $\phi=0.0003$.

\begin{tabular}{lcccc}
\hline \hline Wi & Author & $\left(\eta_{\text {eff }}-\eta_{0}\right) / \eta_{0}$ & $\left(N_{1}-N_{1}^{0}\right) / \dot{\gamma}^{2}$ & $\left(N_{2}-N_{2}^{0}\right) / \dot{\gamma}^{2}$ \\
\hline 0.0175 & Escott & $7.580 \times 10^{-4}$ & $1.516 \times 10^{-5}$ & $-1.488 \times 10^{-6}$ \\
& Yang & $7.397 \times 10^{-4}$ & $1.464 \times 10^{-5}$ & $-1.255 \times 10^{-6}$ \\
0.0350 & Escott & $7.580 \times 10^{-4}$ & $3.032 \times 10^{-5}$ & $-2.975 \times 10^{-6}$ \\
& Yang & $7.397 \times 10^{-4}$ & $2.932 \times 10^{-5}$ & $-2.441 \times 10^{-6}$ \\
0.0700 & Escott & $7.580 \times 10^{-4}$ & $6.064 \times 10^{-5}$ & $-5.951 \times 10^{-6}$ \\
& Yang & $7.365 \times 10^{-4}$ & $5.760 \times 10^{-5}$ & $-4.484 \times 10^{-6}$ \\
\hline \hline
\end{tabular}

which match the normal stress differences given in Eqs. (72) and (73) of Ref. [29] in the dilute limit. In dimensional terms, we calculate

$$
N_{1, \text { dim }}=-2 \alpha_{0, \text { eff }} \dot{\gamma}_{\text {dim }}^{2}, \quad N_{2, \text { dim }}=\left(\alpha_{1, \text { eff }}+2 \alpha_{0, \text { eff }}\right) \dot{\gamma}_{\text {dim }}^{2},
$$

for $\dot{\gamma}_{\text {dim }}$ the dimensional shear rate.

There are numerical results in the literature introduced by Yang et al. [47] at the dilute end of solid volume fraction, and a comparison of our results and theirs are shown in Table III. The $N_{1}^{0}$ and $N_{2}^{0}$ presented in the table are the values of first and second normal stress difference in the absence of a solid phase. The numerical simulation assumes a Giesekus background fluid, with domain a cube and a sphere of diameter $D_{0}$ at its center, with each edge of the cube having a length between $12 D_{0}$ and $24 D_{0}$. We consider the cube with shorter sides of length $12 D_{0}$, which will give a larger solid volume fraction, although admittedly it is still very small at $\phi=0.0003$. The results in Table III show a clear correlation, both in terms of magnitude and sign, with a largest difference between any two results of $25 \%$ at the higher end of $\mathrm{Wi}$. In all cases, the difference from a material function to its value in purely fluid phase is expected to be orders of magnitude smaller than the value, since we are in the dilute limit. Nevertheless, the low percentage difference suggests that a cell model approach as outlined in this paper can predict the behavior of a suspension in viscoelastic fluid, for at least low solid volume fraction.

The components of first and second normal stress difference, normalized by $\dot{\gamma}^{2} \mathrm{Wi}$ are shown in Fig. 7 at $\phi=0.01$ and compared against those presented in Ref. [47]. The total values of $N_{1}$ and $\mathrm{N}_{2}$ come directly from Eq. (71), and their components are stress in the absence of particles, extra mean polymer stress and stresslet contributions shown in northwest stripes, northeast stripes, and crosshatch, respectively. These terms correspond to $4 \epsilon \mathbf{E}^{\infty \cdot \boldsymbol{\Omega}^{\infty}}+4(1+\epsilon) \overline{\mathbf{E}^{\infty} \cdot \mathbf{E}^{\infty}}, 4 \epsilon\left\langle\widehat{\mathbf{E}^{\prime} \cdot \mathbf{\Omega}^{\prime}}\right\rangle+$ $4(1+\epsilon)\left\langle\widehat{\mathbf{E}^{\prime} \cdot \mathbf{E}^{\prime}}\right\rangle$ and $3 \phi \widehat{\mathbf{S}}^{1} / 4 \pi$ from our analysis in Sec. IID. We can see that the first normal stress difference is dominated by its term in the absence of a solid phase, the only other nonzero contribution being from the stresslet. This agrees with the analysis performed by Koch and Subramanian [30], who showed via a general argument that $N_{1}$ can only be influenced by terms with a dependence on $\boldsymbol{\Omega}^{\infty}$, which both $\left\langle\widehat{E_{i k}^{\prime} \Omega_{k j}^{\prime}}\right\rangle$ and $\left\langle\widehat{E_{i k}^{\prime} E_{k j}^{\prime}}\right\rangle$ do not. The precise values for these two ensemble averages are provided, along with the stresslet contribution in Appendix D.

We also observe that $N_{2} / \dot{\gamma}^{2}$ Wi in total differs very little from its value without a solid phase. Both the extra mean polymer stress term and stresslet terms are nonzero, have roughly the same magnitude at both values of $\phi$ but crucially increase and decrease the stress difference, respectively. These two competing terms overall provide a small negative contribution, which increases the magnitude of the normalized stress difference as a whole by approximately $10 \%$.

Figure 7 also shows the work of Yang et al. [47], who provides the same component breakdown for a numerically based investigation with a Giesekus suspending fluid at $\phi=0.01$. Qualitatively, the analytical and numerical results show very similar characteristics, in most cases differing only in magnitude. Both our component graphs show a dominant bulk fluid stress term to normalized 


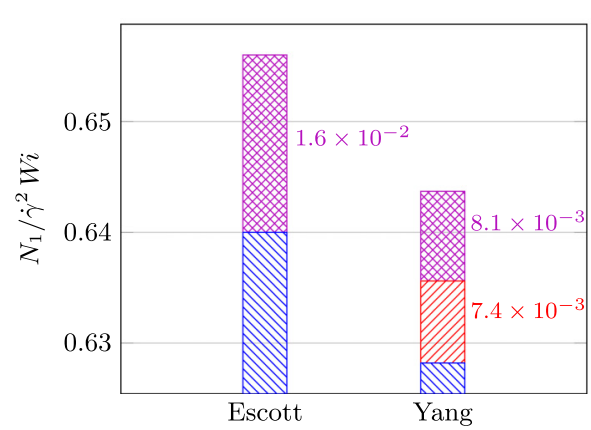

(a)

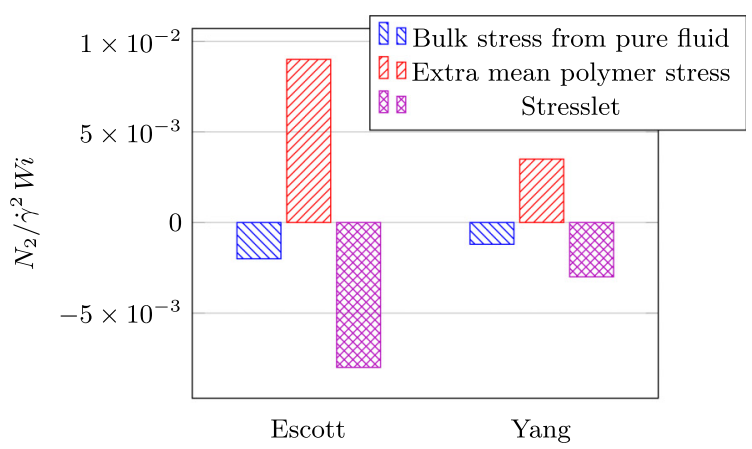

(b)

FIG. 7. The individual components to first and second bulk normal stress differences at solid volume fraction $\phi=0.01$ from our analysis and from Yang et al. [47] are shown in panels (a) and (b), respectively. The normalized $N_{1}$ is presented as a cumulative total, whereas $N_{2}$ directly shows the sizes of each individual term. The northwest shaded bar shows the stress contribution from the fluid phase in the absence of particles, the northeast shaded shows the extra mean polymer stress contribution due to the solid phase and the crosshatched bar shows the stresslet term. We note here that the bulk stress contribution (northwest shaded) in panel (a) is the dominant term, with its value given directly from the $y$ axis. The actual values of the extra mean polymer stress and stresslet are shown in the labels to the right of their respective bars. All components are normalized by $\dot{\gamma}^{2}$ Wi with $\epsilon=-0.3198$ and -0.500977 for $N_{1}$ and $N_{2}$, respectively. The inertial stress terms present in Ref. [47] have been omitted from both charts.

$N_{1}$ and an overall negative $N_{2}$ value which is small in magnitude. Both investigations also show competing extra mean polymer stress and stresslet terms for $N_{2}$, in which the extra mean polymer stress term provides slightly more. As aforementioned, the magnitude of the second normal stress differences are apart by approximately $60 \%$, and also the stresslet contribution to $N_{1}$ is twice the value of its numerical counterpart. Further, there is a nonzero contribution from the extra polymer stress in [47] to $N_{1}$ which we predict to be zero. While there are distinct differences between the two, we should note that the numerical analysis was taken at $\mathrm{Wi}=1$ which violates our assumption that $\mathrm{Wi}$ is a small perturbation parameter, so we do not expect there to be an exact correlation between the two results. However, we consider the qualitative agreement between the results a good step towards validating the cell model approach.

We scale these normal stress differences with the shear stress $\sigma_{x y}$, and find

$$
\frac{N_{1}}{\sigma_{x y}}=-2 \dot{\gamma} \epsilon \mathrm{Wi}, \quad \frac{N_{2}}{\sigma_{x y}}=\dot{\gamma}\left(\frac{\alpha_{1, \mathrm{eff}}}{\alpha_{0}} \frac{\eta_{0}}{\eta_{\mathrm{eff}}}+2 \epsilon\right) \mathrm{Wi},
$$

the second of which is shown in Fig. 8. From the first expression in Eq. (73), it is clear that our first normal stress difference $N_{1}$ can only be positive. Further, as seen in Fig. 8, the second normal stress difference is negative for the large majority of concentrations. However, using the cell model proxy, we predict a change in sign of $N_{2}$ at some solid volume fraction for parameter $\epsilon \in(-7 / 14,-9 / 14)$.

The only choice of solvent parameters for which $N_{2}$ does not change sign is $\epsilon=-0.5$, the socalled Weissenberg fluid. It is defined as a second-order fluid for which there is zero second normal stress difference, i.e., $N_{2}^{0}=0$. In this case, adding particles results in a positive $N_{2} / \sigma_{x y}$ which, as seen in Fig. 8, will increase with concentration and diverge as it approaches packing.

Yang et al. [47] performed a numerical investigation of the second normal stress difference in dilute suspensions over a range of Weissenberg numbers, which they compared against the theory provided by Ref. [30]. We show the results from both papers at Weissenberg number $\mathrm{Wi}=0.1$ and our semianalytic solution for the same value of $\epsilon$ in Fig 9. We see that there is a good correlation between the results, both qualitatively and quantitatively; there is a change in sign for all results 


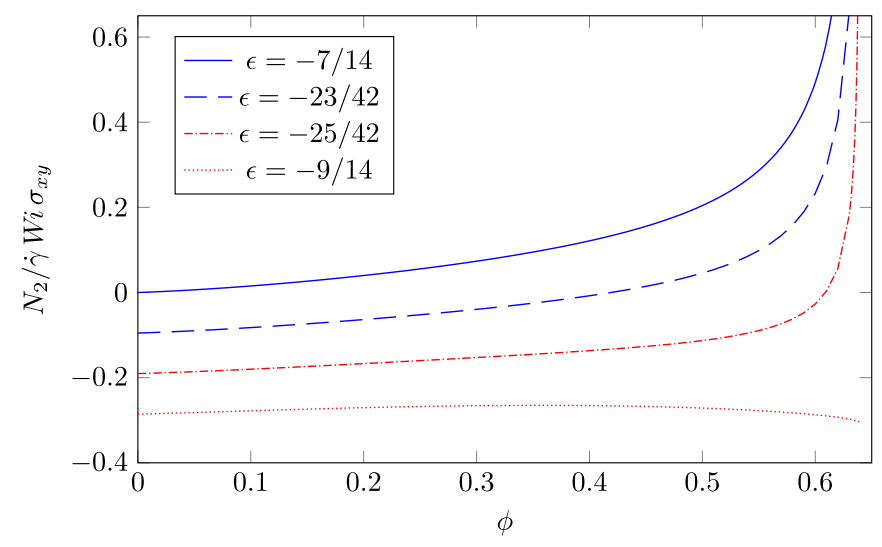

FIG. 8. The second normal stress difference $N_{2}$ scaled by $\dot{\gamma}$, Wi, and $\sigma_{x y}$, the shear rate, Weissenberg number, and shear stress, respectively. The four choices of parameter $\epsilon$ are $-7 / 14$ the solid line, $-23 / 42$ the dashed line, $-25 / 42$ the dash dotted line and $-9 / 14$ the dotted line. It shows the scaled $N_{2}$ as a function of solid volume fraction $\phi$ using our Krieger-Dougherty matching $b(\phi)$, having taken $\phi_{m}=0.64$ as the maximum random packing fraction for monodisperse spheres.

in the range of concentration $\phi \in[0.015,0.025]$. In particular, our results and those of Yang et al. [47] are in good agreement, both showing a change of sign at roughly $\phi=0.0175$, a linear trend for increasing solid volume fraction and similar negative values in the absence of solid particles.

A comparison between our calculated formulas for $N_{1}$ and $N_{2}$ in suspension and the experimental values found in Dai et al. [15] is given in Fig. 10. The experiments were completed for a suspension of solid spheres in a Boger background fluid, with a suspending viscosity of $2.16 \mathrm{~Pa}$ s, mean particle diameter $42.3 \mu \mathrm{m}$ and standard deviation of $0.63 \mu \mathrm{m}$ in size. We observe that in both Figs. 10(a) and 10(b), our analytical solutions have a close matching with Ref. [15] up to approximately $\phi=0.25$. At this point, the behavior of the experimental values drastically changes and grows exponentially in magnitude in the negative direction. However, our equations for normal stress differences given in Eq. (71) remain positive (for $\epsilon=-1 / 2$ ) and do not show any clear exponential behavior in the range of solid volume fraction given.

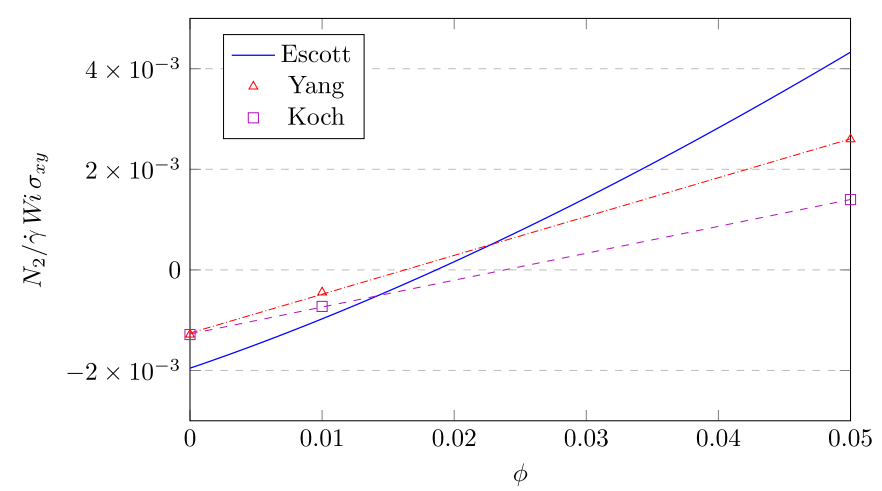

FIG. 9. The second normal stress difference $N_{2}$ scaled by $\dot{\gamma}$, Wi, and $\sigma_{x y}$, the shear rate, Weissenberg number, and shear stress, respectively. The results of Escott and Wilson are shown against the data points from Yang et al. [47] and Koch and Subramanian [30] for three values of solid volume fraction, along with their respective linear regression lines. The value of $\epsilon$ which matches that of [47] is $\epsilon=-0.500977$. 


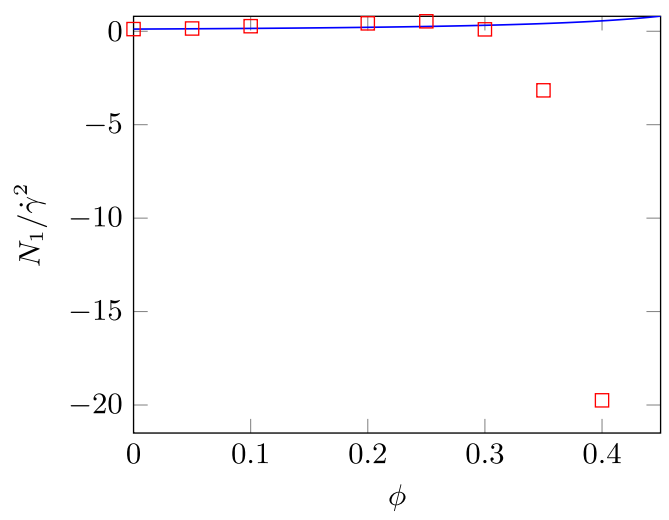

(a)

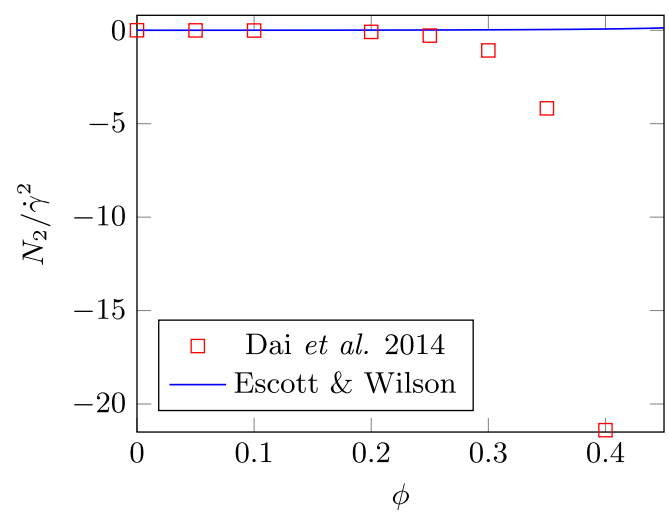

(b)

FIG. 10. The first and second normal stress differences from our analytics and the experiments of Dai et al. [15] are given in panels (a) and (b), respectively. $N_{1}$ and $N_{2}$ are scaled with $\dot{\gamma}^{2}$, and shown over a range of solid volume fraction up to $\phi=0.4$. We consider the choice of parameter $\epsilon=-1 / 2$ based on the ratio $N_{2} / N_{1}=0$ at $\phi=0$ found experimentally [15]. Our Weissenberg number here is $\mathrm{Wi}=0.116$ based on the value of $N_{1}$ in the absence of a solid phase.

The cell model approach has provided us with Eqs. (71) for $N_{1}$ and $N_{2}$ which show similar behavior to experiments up to approximately $\phi=0.25$. The clear difference in both qualitative and quantitative behavior after this point highlights the limits of the cell model in dense suspensions, where solid mechanics become far more important to viscometric functions. Such physical considerations as particle contacts and inertia have been neglected in this model, which even in Newtonian suspending fluid, play a vital role to the viscometric functions. As shown in Figs. 10, their contribution becomes dominant past a certain concentration, which causes the clear disparity between our solutions and the experimental data [15].

\section{E. Which interparticle distance is appropriate?}

Whilst we have engineered a decoupling of $b$ and $\phi$ with the intention of matching effective viscosity to the Krieger-Dougherty equation, we could elect to use the mean interparticle distance instead. In our case, assuming that the mean distance between particles $d$ is measured from surface to surface, the correct choice of cell radius is $b=1+d / 2$. It is notoriously difficult in experimentation to measure the mean distance between particles in a fluid suspension, and recent work has gone into its measurement. Park [48] has developed an image processing method for measuring the mean interparticle distance and applied it to two large choices of solid volume fraction $\phi=0.4$ and 0.5 . We have taken these data points and converted them to values of the nondimensionalised cell radius. We then calculated the bulk material functions at these two values of $\phi$, along with the percentage difference between the two, and the results are shown in Table IV. We choose $\epsilon=-23 / 42$ as it is within the parameter range detailed in Sec. IV D, and the normalized $N_{2}$ changes sign at this value between $\phi=0.4$ and 0.5 as shown in Fig. 8.

The value of cell radius $b$ found using the mean interparticle distance underpredicts our numerical matching at both discrete values of concentration, which results in an increase in effective viscosity. We also see that, at these high solid volume fractions, the difference between $b$ using the Krieger-Dougherty matching and the optical method in Ref. [48] is relatively small, at most $11.39 \%$ for our discrete concentrations. However, it is clear that this small disparity between the values causes a large change in the effective viscosity and bulk normal stress differences, the maximum difference being in second normal stress difference at $\phi=0.4$. Here we see that $N_{2}$ using our Krieger-Dougherty matching defined in Eq. (49) is less than a sixth the magnitude of 
TABLE IV. Values for $b$, bulk material functions and their normalized counterparts using the KriegerDougherty matching and mean interparticle distance in the measure of cell volume. We take $\phi_{m}=0.64, \epsilon=$ $-23 / 42$ and normalize by $\sigma_{x y}$. Note that we define $\%$ difference of two numbers $x$ and $y$ as $\% \operatorname{Diff}(x, y)=$ $|(x-y) / \operatorname{Max}[x, y]|$.

\begin{tabular}{llcccccc}
\hline \hline$\phi$ & \multicolumn{1}{c}{ Method } & $b$ & $\eta_{\text {eff }} / \eta_{0}$ & $N_{1} / \dot{\gamma}^{2} \mathrm{Wi}$ & $N_{1} / \dot{\gamma} \mathrm{Wi} \sigma_{x y}$ & $N_{2} / \dot{\gamma}^{2} \mathrm{Wi}$ & $N_{2} / \dot{\gamma} \mathrm{Wi} \sigma_{x y}$ \\
\hline 0.4 & Krieger-Dougherty matching & 1.602 & 4.803 & 5.261 & 1.095 & -0.037 & -0.008 \\
& Mean interparticle distance & 1.420 & 8.097 & 8.868 & 1.095 & 0.255 & 0.032 \\
& \% Difference & 11.39 & 40.68 & 40.68 & 0 & 114.4 & 124.3 \\
0.5 & Krieger-Dougherty matching & 1.387 & 11.38 & 12.46 & 1.095 & 0.519 & 0.046 \\
& Mean interparticle distance & 1.313 & 17.02 & 18.64 & 1.095 & 1.338 & 0.079 \\
& \% Difference & 5.303 & 33.14 & 33.14 & 0 & 61.20 & 41.96 \\
\hline \hline
\end{tabular}

that we calculate based on mean interparticle distance, and also changes sign. Its expected that the normalized $N_{1}$ should have no difference between the two choices of $b$, as can be seen directly by Eq. (73).

There is available experimental data from Dai et al. [15] giving the effective viscosity for a suspension of spherical particles in Boger suspending fluid. The solid volume fraction varies from 0 to 0.4 at intervals of 0.5 or 0.1 , so we can directly compare the two predictions against the data at their most jammed concentration. The one data point is given by their zero shear rate viscosity at $\phi=0.4$, which is estimated from the results at lowest shear rates tested. Based on a suspending viscosity of $2.16 \mathrm{~Pa} \mathrm{~s}$, calculated at $\dot{\gamma}=3.16 \mathrm{~s}^{-1}$, the relative viscosity of the suspension in Ref. [15] at $\phi=0.4$ is 22.14 . This figure is larger than both predictions, approximately by a factor of 5 and 2.5 for the Krieger-Dougherty matching and mean interparticle distance methods, respectively. Whilst the latter prediction is closer to the experimental value, both predictions have a percentage difference larger than $60 \%$. This is another example demonstrating the limitations of the cell model approach in more packed suspensions. The disparity between the experimental value [15] and both calculated values of $\eta_{\text {eff }} / \eta_{0}$ is due to contributions from inertial effects and particle contacts, which we have either neglected in the formulation or been unable to capture with a cell model approach. In our analysis at $\phi=0.4$, either $63 \%$ or $78 \%$ of the effective viscosity, using the empirical $b(\phi)$ and mean interparticle distance, respectively, comes from processes which are not purely hydrodynamic.

The normalized second normal stress difference, given in the final column of Table IV is directly comparable to Fig. 8. The values for Krieger-Dougherty matching are as expected and change sign between our two discrete solid volume fractions. However, what we see from the same calculation using the mean interparticle distances in Ref. [48] are two positive values for $N_{2} / \dot{\gamma}$ Wi $\sigma_{x y}$, both greater in magnitude. This suggests that the normalized $N_{2}$ using the values for $b$ from Ref. [48] does indeed change sign, but at a solid volume fraction lower than predicted by our matching approach.

At the dilute end of the concentration, there is a direct comparison to be made with the work of Yang et al. [47] who considered a lattice approach to numerical simulation using a Giesekus suspending fluid. We have given a comparison already with this data in Table III using the empirical $b(\phi)$ function at solid volume fraction $\phi=0.0003$. The alternative option is to take $b$ defined by the mean interparticle distance $d$, which given that the suspension is very dilute, will be the distance between two particles. Since we consider the cubic lattice in Ref. [47] to have sides of length $12 D_{0}$, with $D_{0}$ diameter of the particle, our corresponding nondimensional value of $b$ will be 12 . The percentage difference between the methods after calculating $\left(\eta_{\text {eff }}-\eta_{0}\right) / \eta_{0},\left(N_{1}-N_{1}^{0}\right) / \dot{\gamma}^{2}$ and $\left(N_{2}-N_{2}^{0}\right) / \dot{\gamma}^{2}$ were $0.3 \%, 0.3 \%$ and $0.08 \%$, respectively.

These results are expected given that we are in the dilute region. The actual values of the extra contributions to viscometric functions above are exceptionally similar between methods of choosing $b$, to such a degree that the differences are $\mathrm{O}\left(10^{-5}\right)$ at maximum. Nevertheless, we find that for both the effective viscosity and first normal stress difference, the mean interparticle distance method has 
a greater disparity with the numerical data [47]. In the case of $N_{2}$ however, its prediction is closer to the numerical value than that of the Krieger-Dougherty matching process.

\section{CONCLUSIONS}

We have investigated in this paper the rheology of a suspension of solid spheres in a second-order fluid, using a cell model to capture the effects of finite solid concentration. We provide the general result for all steady linear background flows and evaluate the rheology in a simple shear flow.

Using an empirical method to determine the appropriate cell volume at any volume fraction, we can match the viscosity to the Krieger-Dougherty relation and deduce the behavior of the other material functions. The macroscopic behavior of the suspension is thus characterised as a secondorder fluid itself, with material parameters adjusted for volume fraction of solids. We find that the first normal stress parameter scales exactly as the Krieger-Dougherty expression: that is, the ratio of $N_{1}$ to shear viscosity for the suspension is the same as for the background fluid. The second normal stress difference, however, which is typically negative for the matrix fluid, can become positive as the solid volume fraction is increased.

In the case of a dilute suspension, our predictions for viscometric functions in a simple shear flow coincide precisely with the correct theoretical values [30]. We have shown in Sec. III B that our predictions for effective viscosity at semidilute solid volume fractions $\phi=0.05$ and 0.1 improve on the dilute theory [30] under comparison with the numerical work of Yang and Shaqfeh [43]. At larger values of solid volume fraction, we have made comparisons between our work and experiments [15], for two methods of deriving the cell volume. Both the Krieger-Dougherty matching and mean interparticle distance methods fall short of the observed experimental value by more than $60 \%$. A better prediction is achieved by using the mean interparticle distance in finding the cell volume; however, it is well known that capturing this value experimentally is extremely difficult.

Our method is an improvement on dilute theory, but as the concentration increases, so does the error, and we have no way to bound the error mathematically. Despite this, the method gives a useful enhancement to purely dilute models.

\section{ACKNOWLEDGMENT}

We acknowledge financial support from EPSRC, Grant No. EP/N024915/1.

\section{APPENDIX A: DIFFERENTIAL EQUATIONS GOVERNING THE SCALAR FUNCTIONS FOR A SECOND-ORDER FLUID}

The differential equations governing the scalar velocity and pressure functions $V_{k}(r)$ and $P_{k}(r)$ as defined in Eq. (55) are

$$
\begin{aligned}
r V_{1}{ }^{\prime}(r)+7 V_{1}(r)+\frac{V_{4}{ }^{\prime}(r)}{r} & =0, \\
r V_{2}{ }^{\prime}(r)+5 V_{2}(r)+2 V_{4}(r)+\frac{V_{5}{ }^{\prime}(r)}{r} & =0, \\
r V_{3}{ }^{\prime}(r)+3 V_{3}(r)+V_{5}(r) & =0, \\
r V_{9}{ }^{\prime}(r)+5 V_{9}(r)+2 V_{10}(r)+\frac{V_{11}{ }^{\prime}(r)}{r}-\frac{V_{12}{ }^{\prime}(r)}{r} & =0,
\end{aligned}
$$

from the continuity equation,

$$
\begin{aligned}
P_{1}^{\prime \prime}(r)+\frac{10 P_{1}^{\prime}(r)}{r}=Z_{1}(r), \quad P_{2}^{\prime \prime}(r)+\frac{6 P_{2}{ }^{\prime}(r)}{r}+8 P_{1}(r)=Z_{2}(r), \\
P_{3}^{\prime \prime}(r)+\frac{2 P_{3}^{\prime}(r)}{r}+2 P_{2}(r)=Z_{3}(r), \quad P_{6}^{\prime \prime}(r)+\frac{6 P_{6}^{\prime}(r)}{r}=0,
\end{aligned}
$$


from the stress equation, and

$$
\begin{aligned}
& V_{1}^{\prime \prime}(r)+\frac{12 V_{1}{ }^{\prime}(r)}{r}=\frac{P_{1}{ }^{\prime}(r)}{r}-z_{1}(r), \\
& V_{2}^{\prime \prime}(r)+\frac{8 V_{2}^{\prime}(r)}{r}+8 V_{1}(r)=\frac{P_{2}{ }^{\prime}(r)}{r}-z_{2}(r), \\
& V_{3}^{\prime \prime}(r)+\frac{4 V_{3}^{\prime}(r)}{r}+2 V_{2}(r)=\frac{P_{3}{ }^{\prime}(r)}{r}-z_{3}(r), \\
& V_{4}^{\prime \prime}(r)+\frac{8 V_{4}^{\prime}(r)}{r}+8 V_{1}(r)=4 P_{1}(r)-z_{4}(r), \\
& V_{5}^{\prime \prime}(r)+\frac{4 V_{5}{ }^{\prime}(r)}{r}+4 V_{2}(r)+4 V_{4}(r)=2 P_{2}(r)-z_{5}(r), \\
& V_{9}{ }^{\prime \prime}(r)+\frac{8 V_{9}{ }^{\prime}(r)}{r}=\frac{P_{6}{ }^{\prime}(r)}{r}-z_{9}(r), \\
& V_{11}{ }^{\prime \prime}(r)+\frac{4 V_{11}{ }^{\prime}(r)}{r}+2 V_{9}(r)=P_{6}(r)-z_{11}(r), \\
& V_{12}{ }^{\prime \prime}(r)+\frac{4 V_{12}{ }^{\prime}(r)}{r}-2 V_{9}(r)+4 V_{10}(r)=-P_{6}(r)-z_{12}(r),
\end{aligned}
$$

from the momentum equation, in which

$$
\begin{aligned}
Z_{1}(r)= & -\frac{20(79+120 \epsilon) c_{1} c_{2}}{r^{7}}-\frac{28\left[(59+78 \epsilon) c_{1} c_{3}-10(1+2 \epsilon) c_{2} c_{4}\right]}{r^{9}}+\frac{20(6+5 \epsilon) c_{2}^{2}}{r^{12}} \\
& +\frac{72(8+11 \epsilon) c_{2} c_{3}}{r^{14}}+\frac{252(2+3 \epsilon) c_{3}^{2}}{r^{16}}, \\
Z_{2}(r)= & 12(72+143 \epsilon) c_{1}^{2}+\frac{4(227+348 \epsilon) c_{1} c_{2}}{r^{5}}+\frac{16\left[(59+78 \epsilon) c_{1} c_{3}-10(1+2 \epsilon) c_{2} c_{4}\right]}{r^{7}} \\
& +\frac{16(9+11 \epsilon) c_{2}^{2}}{r^{10}}+\frac{96(8+11 \epsilon) c_{2} c_{3}}{r^{12}}+\frac{432(2+3 \epsilon) c_{3}^{2}}{r^{14}}, \\
Z_{3}(r)= & (279+548 \epsilon) c_{1}^{2} r^{2}-\frac{4(23+36 \epsilon) c_{1} c_{2}}{r^{3}}-\frac{8\left[(59+78 \epsilon) c_{1} c_{3}-10(1+2 \epsilon) c_{2} c_{4}\right]}{5 r^{5}} \\
& -84(1+2 \epsilon) c_{1} c_{4}+\frac{4(1+2 \epsilon) c_{2}^{2}}{r^{8}}+\frac{16(8+11 \epsilon) c_{2} c_{3}}{5 r^{10}}
\end{aligned}
$$

and

$$
\begin{aligned}
z_{1}(r)= & -\frac{5(68+109 \epsilon) c_{1} c_{2}}{r^{7}}-\frac{14\left[(52+71 \epsilon) c_{1} c_{3}-10(1+2 \epsilon) c_{2} c_{4}\right]}{r^{9}}-\frac{20(2+\epsilon) c_{2}^{2}}{r^{12}} \\
& -\frac{72(2+3 \epsilon) c_{2} c_{3}}{r^{14}}-\frac{252(2+3 \epsilon) c_{3}^{2}}{5 r^{16}}+\frac{126(2+3 \epsilon) c_{3} c_{4}}{r^{11}}, \\
z_{2}(r)= & 6(13+37 \epsilon) c_{1}^{2}+\frac{2(73+112 \epsilon) c_{1} c_{2}}{r^{5}}+\frac{2\left[(148+201 \epsilon) c_{1} c_{3}-10(3+5 \epsilon) c_{2} c_{4}\right]}{r^{7}} \\
& -\frac{16(2+3 \epsilon) c_{2}^{2}}{r^{10}}-\frac{24(5+7 \epsilon) c_{2} c_{3}}{r^{12}}-\frac{228(2+3 \epsilon) c_{3}^{2}}{5 r^{14}}-\frac{56(2+3 \epsilon) c_{3} c_{4}}{r^{9}}, \\
z_{3}(r)= & 5(3+8 \epsilon) c_{1}^{2} r^{2}-\frac{10(1+\epsilon) c_{1} c_{2}}{r^{3}}-\frac{2\left[(44+59 \epsilon) c_{1} c_{3}-10(1+\epsilon) c_{2} c_{4}\right]}{5 r^{5}}-2(3+8 \epsilon) c_{1} c_{4} \\
& -\frac{8(1+\epsilon) c_{2} c_{3}}{5 r^{10}}-\frac{8(2+3 \epsilon) c_{3}^{2}}{5 r^{12}}+\frac{4(2+3 \epsilon) c_{3} c_{4}}{r^{7}},
\end{aligned}
$$




$$
\begin{aligned}
z_{4}(r)= & 3(11-15 \epsilon) c_{1}^{2}+\frac{4(79+120 \epsilon) c_{1} c_{2}}{r^{5}}+\frac{4\left[(111+149 \epsilon) c_{1} c_{3}-20(1+2 \epsilon) c_{2} c_{4}\right]}{r^{7}}+\frac{8 c_{2}^{2}}{r^{10}} \\
& +\frac{12(3+5 \epsilon) c_{2} c_{3}}{r^{12}}+\frac{72(2+3 \epsilon) c_{3}^{2}}{5 r^{14}}-\frac{56(2+3 \epsilon) c_{3} c_{4}}{r^{9}}, \\
z_{5}(r)= & 12(17+29 \epsilon) c_{1}^{2} r^{2}-\frac{4(23+36 \epsilon) c_{1} c_{2}}{r^{3}}-\frac{4\left[(162+215 \epsilon) c_{1} c_{3}-5(3+5 \epsilon) c_{2} c_{4}\right]}{5 r^{5}} \\
& +6(11+20 \epsilon) c_{1} c_{4}+\frac{4(1+2 \epsilon) c_{2}^{2}}{r^{8}}+\frac{24(3+5 \epsilon) c_{2} c_{3}}{5 r^{10}}+\frac{48(2+3 \epsilon) c_{3}^{2}}{5 r^{12}}+\frac{16(2+3 \epsilon) c_{3} c_{4}}{r^{7}}, \\
z_{9}(r)= & -\frac{20 \epsilon c_{2}}{r^{7}}, \quad z_{11}(r)=-21 \epsilon c_{1}+\frac{4 \epsilon c_{2}}{r^{5}}, \quad z_{12}(r)=21 \epsilon c_{1}-\frac{4 \epsilon c_{2}}{r^{5}},
\end{aligned}
$$

with constants $c_{i}$ given in Eq. (43).

\section{APPENDIX B: EXPLICIT VALUES OF THE CONSTANTS FOUND IN SCALAR \\ FUNCTIONS FOR A SECOND-ORDER FLUID}

The constants $C_{i}$ found in the second-order velocity and pressure solutions, as introduced in Eq. (55), have the general form

$$
\begin{aligned}
C_{i}= & \mu_{1,1}^{i}(b, \epsilon) c_{1}^{2}+\mu_{1,2}^{i}(b, \epsilon) c_{1} c_{2}+\mu_{1,3}^{i}(b, \epsilon) c_{1} c_{3}+\mu_{1,4}^{i}(b, \epsilon) c_{1} c_{4}+\mu_{2,2}^{i}(b, \epsilon) c_{2}^{2} \\
& +\mu_{2,3}^{i}(b, \epsilon) c_{2} c_{3}+\mu_{2,4}^{i}(b, \epsilon) c_{2} c_{4}+\mu_{3,3}^{i}(b, \epsilon) c_{3}^{2}+\mu_{3,4}^{i}(b, \epsilon) c_{3} c_{4}+\mu_{4,4}^{i}(b, \epsilon) c_{4}^{2},
\end{aligned}
$$

with constants $c_{i}$ provided in Eq. (43). All values of $\mu$ can be described as

$$
\mu_{a, b}^{i}(b, \epsilon)=\frac{\lambda_{a, b}^{i}(b, \epsilon)}{\kappa^{i}(b)},
$$

for the denominator functions

$$
\begin{aligned}
\kappa^{1}(b)= & b\left(4+16 b+40 b^{2}+80 b^{3}+140 b^{4}+224 b^{5}+336 b^{6}+399 b^{7}+336 b^{8}+224 b^{9}\right. \\
& \left.+140 b^{10}+80 b^{11}+40 b^{12}+16 b^{13}+4 b^{14}\right) \\
\kappa^{2}(b)= & \kappa^{7}(b)=\kappa^{8}(b)=b^{2} \kappa^{1}(b) \\
\kappa^{3}(b)= & \frac{\kappa^{4}(b)}{b^{2}}=b^{2}\left(4+16 b+40 b^{2}+55 b^{3}+40 b^{4}+16 b^{5}+4 b^{6}\right) \kappa^{1}(b) \\
\kappa^{5}(b)= & \kappa^{6}(b)=\kappa^{9}(b)=b^{5}\left(4+16 b+40 b^{2}+55 b^{3}+40 b^{4}+16 b^{5}+4 b^{6}\right) \\
\kappa^{10}(b)= & \kappa^{11}(b)=\frac{\kappa^{1}(b)}{b} \\
\kappa^{12}(b)= & \kappa^{13}(b)=\kappa^{5}(b),
\end{aligned}
$$

and all nonzero values of $\lambda$ :

$$
\begin{aligned}
\lambda_{1,2}^{1}(b, \epsilon)= & -\frac{121 b^{7}}{2}\left(8+32 b+80 b^{2}+125 b^{3}+140 b^{4}+125 b^{5}+80 b^{6}+32 b^{7}+8 b^{8}\right)(1+\epsilon), \\
\lambda_{1,3}^{1}(b, \epsilon)= & -\frac{693 b^{5}}{10}\left(4+16 b+40 b^{2}+80 b^{3}+140 b^{4}+175 b^{5}\right. \\
& \left.+140 b^{6}+80 b^{7}+40 b^{8}+16 b^{9}+4 b^{10}\right)(1+\epsilon), \\
\lambda_{2,2}^{1}(b, \epsilon)= & 198 b^{2}\left(1+4 b+10 b^{2}+20 b^{3}+35 b^{4}+56 b^{5}+84 b^{6}+84 b^{7}+56 b^{8}+35 b^{9}\right. \\
& \left.+20 b^{10}+10 b^{11}+4 b^{12}+b^{13}\right)(1+\epsilon),
\end{aligned}
$$




$$
\begin{aligned}
& \lambda_{2,3}^{1}(b, \epsilon)=-\frac{1188}{5}\left(1+4 b+10 b^{2}+20 b^{3}+35 b^{4}+56 b^{5}+84 b^{6}+105 b^{7}+105 b^{8}+84 b^{9}\right. \\
& \left.+56 b^{10}+35 b^{11}+20 b^{12}+10 b^{13}+4 b^{14}+b^{15}\right)(1+\epsilon), \\
& \lambda_{1,2}^{2}(b, \epsilon)=-\frac{22 b^{3}}{3}\left(1+4 b+10 b^{2}+20 b^{3}+28 b^{4}+28 b^{5}+20 b^{6}+10 b^{7}+4 b^{8}+b^{9}\right)(1+\epsilon), \\
& \lambda_{1,3}^{2}(b, \epsilon)=-\frac{14 b^{3}}{5}\left(5+20 b+36 b^{2}+44 b^{3}+44 b^{4}+36 b^{5}+20 b^{6}+5 b^{7}\right)(1+\epsilon), \\
& \lambda_{2,2}^{2}(b, \epsilon)=2 b^{2}\left(7+12 b+15 b^{2}+16 b^{3}+15 b^{4}+12 b^{5}+7 b^{6}\right)(1+\epsilon), \\
& \lambda_{2,3}^{2}(b, \epsilon)=\frac{4}{5}\left(7+28 b+43 b^{2}+52 b^{3}+55 b^{4}+52 b^{5}+43 b^{6}+28 b^{7}+7 b^{8}\right)(1+\epsilon), \\
& \lambda_{1,1}^{3}(b, \epsilon)=35 b^{8}\left(3+12 b+20 b^{2}+20 b^{3}+12 b^{4}+3 b^{5}\right)\left(4+16 b+40 b^{2}+80 b^{3}\right. \\
& +140 b^{4}+224 b^{5}+336 b^{6}+339 b^{7}+336 b^{8}+224 b^{9}+140 b^{10}+80 b^{11} \\
& \left.+40 b^{12}+16 b^{13}+4 b^{14}\right)(1+\epsilon), \\
& \lambda_{1,2}^{3}(b, \epsilon)=-b^{7}\left(2544+16212 b+58464 b^{2}+153800 b^{3}+323200 b^{4}+565996 b^{5}\right. \\
& +853668 b^{6}+1113396 b^{7}+1223215 b^{8}+1113396 b^{9}+853668 b^{10}+565996 b^{11} \\
& \left.+323200 b^{12}+153800 b^{13}+58464 b^{14}+16212 b^{15}+2544 b^{16}\right)(1+\epsilon), \\
& \lambda_{1,3}^{3}(b, \epsilon)=-\frac{84 b^{5}}{5}\left(4+16 b+40 b^{2}+55 b^{3}+40 b^{4}+16 b^{5}+4 b^{6}\right)\left(8+32 b+80 b^{2}+160 b^{3}\right. \\
& \left.+280 b^{4}+385 b^{5}+420 b^{6}+385 b^{7}+280 b^{8}+160 b^{9}+80 b^{10}+32 b^{11}+8 b^{12}\right)(1+\epsilon), \\
& \lambda_{2,2}^{3}(b, \epsilon)=-16 b^{2}\left(32+256 b+1152 b^{2}+3665 b^{3}+9160 b^{4}+19250 b^{5}+35640 b^{6}+59650 b^{7}\right. \\
& +90440 b^{8}+122258 b^{9}+143597 b^{10}+143597 b^{11}+122258 b^{12}+90440 b^{13}+59650 b^{14} \\
& \left.+35640 b^{15}+19250 b^{16}+9160 b^{17}+3665 b^{18}+1152 b^{19}+256 b^{20}+32 b^{21}\right)(1+\epsilon) \text {, } \\
& \lambda_{2,3}^{3}(b, \epsilon)=-\frac{8}{5}\left(148+1184 b+5328 b^{2}+17060 b^{3}+43240 b^{4}+92820 b^{5}+176400 b^{6}+303555 b^{7}\right. \\
& +476160 b^{8}+677150 b^{9}+864492 b^{10}+980713 b^{11}+980713 b^{12}+864492 b^{13} \\
& +677150 b^{14}+476160 b^{15}+303555 b^{16}+176400 b^{17}+92820 b^{18} \\
& \left.+43240 b^{19}+17060 b^{20}+5328 b^{21}+1184 b^{22}+148 b^{23}\right)(1+\epsilon), \\
& \lambda_{1,1}^{4}(b, \epsilon)=-b^{5}\left(8+32 b+80 b^{2}+125 b^{3}+140 b^{4}+125 b^{5}+80 b^{6}+32 b^{7}+8 b^{8}\right) \\
& \times\left(4+16 b+40 b^{2}+80 b^{3}+140 b^{4}+224 b^{5}+336 b^{6}+399 b^{7}+336 b^{8}+224 b^{9}\right. \\
& \left.+140 b^{10}+80 b^{11}+40 b^{12}+16 b^{13}+4 b^{14}\right)(1+\epsilon) \text {, } \\
& \lambda_{1,2}^{4}(b, \epsilon)=-\frac{2 b^{5}}{63}\left(556+4448 b+10080 b^{2}+2387 b^{3}-38756 b^{4}-114114 b^{5}-170992 b^{6}\right. \\
& -127914 b^{7}-26540 b^{8}-26540 b^{9}-127914 b^{10}-170992 b^{11}-114114 b^{12} \\
& \left.-38756 b^{13}+2387 b^{14}+10080 b^{15}+4448 b^{16}+556 b^{17}\right)(1+\epsilon), \\
& \lambda_{1,3}^{4}(b, \epsilon)=2 b^{5}\left(4+16 b+40 b^{2}+55 b^{3}+40 b^{4}+16 b^{5}+4 b^{6}\right)\left(7+28 b+52 b^{2}+68 b^{3}+76 b^{4}\right. \\
& \left.+76 b^{5}+68 b^{6}+52 b^{7}+28 b^{8}+7 b^{9}\right)(1+\epsilon)
\end{aligned}
$$




$$
\begin{aligned}
& \lambda_{2,2}^{4}(b, \epsilon)=\frac{2 b^{2}}{7}\left(32+256 b+812 b^{2}+1648 b^{3}+2324 b^{4}+3045 b^{5}+5516 b^{6}+11049 b^{7}\right. \\
& +17584 b^{8}+20573 b^{9}+17584 b^{10}+11049 b^{11}+5516 b^{12}+3045 b^{13}+2324 b^{14} \\
& \left.+1648 b^{15}+812 b^{16}+256 b^{17}+32 b^{18}\right)(1+\epsilon) \text {, } \\
& \lambda_{2,3}^{4}(b, \epsilon)=\frac{4}{35}\left(72+576 b+2212 b^{2}+5600 b^{3}+10740 b^{4}+17605 b^{5}+29120 b^{6}+50307 b^{7}\right. \\
& +80176 b^{8}+107819 b^{9}+119196 b^{10}+107819 b^{11}+80176 b^{12}+50307 b^{13}+29120 b^{14} \\
& \left.+17605 b^{15}+10740 b^{16}+5600 b^{17}+2212 b^{18}+576 b^{19}+72 b^{20}\right)(1+\epsilon) \\
& \lambda_{1,1}^{5}(b, \epsilon)=3 b^{8}\left(5+20 b+36 b^{2}+44 b^{3}+44 b^{4}+36 b^{5}+20 b^{6}+5 b^{7}\right)(1+\epsilon), \\
& \lambda_{1,2}^{5}(b, \epsilon)=-\frac{69 b^{7}}{7}\left(8+17 b+20 b^{2}+17 b^{3}+8 b^{4}\right)(1+\epsilon) \\
& \lambda_{1,3}^{5}(b, \epsilon)=-\frac{3 b^{5}}{5}\left(4+16 b+40 b^{2}+55 b^{3}+40 b^{4}+16 b^{5}+4 b^{6}\right)(1+\epsilon), \\
& \lambda_{2,2}^{5}(b, \epsilon)=-\frac{16 b^{2}}{7}\left(1+4 b+10 b^{2}+20 b^{3}+35 b^{4}+35 b^{5}+20 b^{6}+10 b^{7}+4 b^{8}+b^{9}\right)(1+\epsilon), \\
& \lambda_{2,3}^{5}(b, \epsilon)=-\frac{24}{35}\left(3+12 b+30 b^{2}+60 b^{3}+105 b^{4}+140 b^{5}+140 b^{6}+105 b^{7}+60 b^{8}\right. \\
& \left.+30 b^{9}+12 b^{10}+3 b^{11}\right)(1+\epsilon), \\
& \lambda_{1,1}^{6}(b, \epsilon)=\frac{b^{5}}{2}\left(4+16 b+40 b^{2}+80 b^{3}+140 b^{4}+175 b^{5}+140 b^{6}+80 b^{7}\right. \\
& \left.+40 b^{8}+16 b^{9}+4 b^{10}\right)(1+\epsilon), \\
& \lambda_{1,2}^{6}(b, \epsilon)=\frac{46 b^{5}}{7}\left(3+12 b+20 b^{2}+20 b^{3}+12 b^{4}+3 b^{5}\right)(1+\epsilon), \\
& \lambda_{2,2}^{6}(b, \epsilon)=-\frac{8 b^{2}}{21}\left(5+20 b+29 b^{2}+32 b^{3}+29 b^{4}+20 b^{5}+5 b^{6}\right)(1+\epsilon), \\
& \lambda_{2,3}^{6}(b, \epsilon)=-\frac{4}{7}\left(3+12 b+23 b^{2}+32 b^{3}+35 b^{4}+32 b^{5}+23 b^{6}+12 b^{7}+3 b^{8}\right)(1+\epsilon), \\
& \lambda_{1,2}^{7}(b, \epsilon)=-\frac{77 b^{7}}{3}\left(4+16 b+40 b^{2}+65 b^{3}+80 b^{4}+85 b^{5}+80 b^{6}+65 b^{7}\right. \\
& \left.+40 b^{8}+16 b^{9}+4 b^{10}\right)(1+\epsilon), \\
& \lambda_{1,3}^{7}(b, \epsilon)=-\frac{49 b^{5}}{5}\left(8+32 b+80 b^{2}+160 b^{3}+280 b^{4}+385 b^{5}+420 b^{6}+385 b^{7}+280 b^{8}\right. \\
& \left.+160 b^{9}+80 b^{10}+32 b^{11}+8 b^{12}\right)(1+\epsilon), \\
& \lambda_{2,2}^{7}(b, \epsilon)=-28 b^{2}\left(1+4 b+10 b^{2}+20 b^{3}+35 b^{4}+56 b^{5}+84 b^{6}+120 b^{7}+120 b^{8}+84 b^{9}\right. \\
& \left.+56 b^{10}+35 b^{11}+20 b^{12}+10 b^{13}+4 b^{14}+b^{15}\right)(1+\epsilon) \text {, } \\
& \lambda_{2,3}^{7}(b, \epsilon)=-\frac{56}{5}\left(1+4 b+10 b^{2}+20 b^{3}+35 b^{4}+56 b^{5}+84 b^{6}+120 b^{7}+165 b^{8}+165 b^{9}\right. \\
& \left.+120 b^{10}+84 b^{11}+56 b^{12}+35 b^{13}+20 b^{14}+10 b^{15}+4 b^{16}+b^{17}\right)(1+\epsilon),
\end{aligned}
$$




$$
\begin{aligned}
& \lambda_{1,2}^{8}(b, \epsilon)=-\frac{55 b^{3}}{9}\left(5+20 b+50 b^{2}+100 b^{3}+148 b^{4}+172 b^{5}+172 b^{6}+148 b^{7}+100 b^{8}\right. \\
& \left.+50 b^{9}+20 b^{10}+5 b^{11}\right)(1+\epsilon), \\
& \lambda_{1,3}^{8}(b, \epsilon)=-7 b^{3}\left(7+28 b+52 b^{2}+68 b^{3}+76 b^{4}+76 b^{5}+68 b^{6}+52 b^{7}+28 b^{8}+7 b^{9}\right)(1+\epsilon), \\
& \lambda_{2,2}^{8}(b, \epsilon)=5 b^{2}\left(9+16 b+21 b^{2}+24 b^{3}+25 b^{4}+24 b^{5}+21 b^{6}+16 b^{7}+9 b^{8}\right)(1+\epsilon), \\
& \lambda_{2,3}^{8}(b, \epsilon)=6\left(3+12 b+19 b^{2}+24 b^{3}+27 b^{4}+28 b^{5}+27 b^{6}+24 b^{7}+19 b^{8}+12 b^{9}+3 b^{10}\right)(1+\epsilon), \\
& \lambda_{1,1}^{9}(b, \epsilon)=-\frac{3 b^{5}}{2}\left(4+16 b+40 b^{2}+80 b^{3}+140 b^{4}+175 b^{5}+140 b^{6}+80 b^{7}\right. \\
& \left.+40 b^{8}+16 b^{9}+4 b^{10}\right)(1+\epsilon), \\
& \lambda_{1,2}^{9}(b, \epsilon)=-\frac{138 b^{5}}{7}\left(3+12 b+20 b^{2}+20 b^{3}+12 b^{4}+3 b^{5}\right)(1+\epsilon), \\
& \lambda_{2,2}^{9}(b, \epsilon)=\frac{8 b^{2}}{7}\left(5+20 b+29 b^{2}+32 b^{3}+29 b^{4}+20 b^{5}+5 b^{6}\right)(1+\epsilon), \\
& \lambda_{2,3}^{9}(b, \epsilon)=\frac{12}{7}\left(3+12 b+23 b^{2}+32 b^{3}+35 b^{4}+32 b^{5}+23 b^{6}+12 b^{7}+3 b^{8}\right)(1+\epsilon), \\
& \lambda_{1,2}^{10}(b, \epsilon)=231 b^{4}\left(4+16 b+40 b^{2}+65 b^{3}+80 b^{4}+85 b^{5}+80 b^{6}+65 b^{7}\right. \\
& \left.+40 b^{8}+16 b^{9}+4 b^{10}\right)(1+\epsilon), \\
& \lambda_{1,3}^{10}(b, \epsilon)=\frac{441 b^{2}}{5}\left(8+32 b+80 b^{2}+160 b^{3}+280 b^{4}+385 b^{5}+420 b^{6}+385 b^{7}+280 b^{8}\right. \\
& \left.+160 b^{9}+80 b^{10}+32 b^{11}+8 b^{12}\right)(1+\epsilon), \\
& \lambda_{2,2}^{10}(b, \epsilon)=\frac{252}{b}\left(1+4 b+10 b^{2}+20 b^{3}+35 b^{4}+56 b^{5}+84 b^{6}+120 b^{7}+120 b^{8}+84 b^{9}\right. \\
& \left.+56 b^{10}+35 b^{11}+20 b^{12}+10 b^{13}+4 b^{14}+b^{15}\right)(1+\epsilon), \\
& \lambda_{2,3}^{10}(b, \epsilon)=\frac{504}{5 b^{3}}\left(1+4 b+10 b^{2}+20 b^{3}+35 b^{4}+56 b^{5}+84 b^{6}+120 b^{7}+165 b^{8}+165 b^{9}\right. \\
& \left.+120 b^{10}+84 b^{11}+56 b^{12}+35 b^{13}+20 b^{14}+10 b^{15}+4 b^{16}+b^{17}\right)(1+\epsilon), \\
& \lambda_{3,4}^{10}(b, \epsilon)=126\left(4+16 b+40 b^{2}+80 b^{3}+140 b^{4}+224 b^{5}+336 b^{6}+399 b^{7}+336 b^{8}+224 b^{9}\right. \\
& \left.+140 b^{10}+80 b^{11}+40 b^{12}+16 b^{13}+4 b^{14}\right)(2+3 \epsilon) \text {, } \\
& \lambda_{1,1}^{11}(b, \epsilon)=\frac{3}{4}\left(4+16 b+40 b^{2}+80 b^{3}+140 b^{4}+224 b^{5}+336 b^{6}+339 b^{7}+336 b^{8}+224 b^{9}\right. \\
& \left.+140 b^{10}+80 b^{11}+40 b^{12}+16 b^{13}+4 b^{14}\right)(11-15 \epsilon), \\
& \lambda_{1,2}^{11}(b, \epsilon)=\frac{605}{6}\left(1+4 b+10 b^{2}+20 b^{3}+28 b^{4}+28 b^{5}+20 b^{6}+10 b^{7}+4 b^{8}+b^{9}\right)(1+\epsilon), \\
& \lambda_{1,3}^{11}(b, \epsilon)=\frac{77}{2}\left(5+20 b+36 b^{2}+44 b^{3}+44 b^{4}+36 b^{5}+20 b^{6}+5 b^{7}\right)(1+\epsilon), \\
& \lambda_{2,2}^{11}(b, \epsilon)=-\frac{55}{2 b}\left(7+12 b+15 b^{2}+16 b^{3}+15 b^{4}+12 b^{5}+7 b^{6}\right)(1+\epsilon), \\
& \lambda_{2,3}^{11}(b, \epsilon)=-\frac{11}{b^{3}}\left(7+28 b+43 b^{2}+52 b^{3}+55 b^{4}+52 b^{5}+43 b^{6}+28 b^{7}+7 b^{8}\right)(1+\epsilon), \\
& \lambda_{1,1}^{12}(b, \epsilon)=-30 b^{8}\left(5+20 b+36 b^{2}+44 b^{3}+44 b^{4}+36 b^{5}+20 b^{6}+5 b^{7}\right)(1+\epsilon),
\end{aligned}
$$




$$
\begin{aligned}
\lambda_{1,2}^{12}(b, \epsilon)= & \frac{690 b^{7}}{7}\left(8+17 b+20 b^{2}+17 b^{3}+8 b^{4}\right)(1+\epsilon) \\
\lambda_{1,3}^{12}(b, \epsilon)= & 2 b^{5}\left(4+16 b+40 b^{2}+55 b^{3}+40 b^{4}+16 b^{5}+4 b^{6}\right)(172+225 \epsilon) \\
\lambda_{2,2}^{12}(b, \epsilon)= & \frac{160 b^{2}}{7}\left(1+4 b+10 b^{2}+20 b^{3}+35 b^{4}+35 b^{5}+20 b^{6}+10 b^{7}+4 b^{8}+b^{9}\right)(1+\epsilon), \\
\lambda_{2,3}^{12}(b, \epsilon)= & \frac{48}{7}\left(3+12 b+30 b^{2}+60 b^{3}+105 b^{4}+140 b^{5}+140 b^{6}+105 b^{7}+60 b^{8}\right. \\
& \left.+30 b^{9}+12 b^{10}+3 b^{11}\right)(1+\epsilon) \\
\lambda_{2,4}^{12}(b, \epsilon)= & -20 b^{5}\left(4+16 b+40 b^{2}+55 b^{3}+40 b^{4}+16 b^{5}+4 b^{6}\right)(3+5 \epsilon) \\
\lambda_{1,1}^{13}(b, \epsilon)= & \frac{21 b^{5}}{2}\left(8+32 b+80 b^{2}+125 b^{3}+140 b^{4}+125 b^{5}+80 b^{6}+32 b^{7}+8 b^{8}\right)(1+\epsilon), \\
\lambda_{1,2}^{13}(b, \epsilon)= & 138 b^{5}\left(1+4 b+4 b^{2}+b^{3}\right)(1+\epsilon) \\
\lambda_{1,4}^{13}(b, \epsilon)= & -3 b^{5}\left(4+16 b+40 b^{2}+55 b^{3}+40 b^{4}+16 b^{5}+4 b^{6}\right)(11+20 \epsilon) \\
\lambda_{2,2}^{13}(b, \epsilon)= & -24 b^{2}\left(1+4 b+5 b^{2}+4 b^{3}+b^{4}\right)(1+\epsilon), \\
\lambda_{2,3}^{13}(b, \epsilon)= & -\frac{12}{5}\left(9+36 b+65 b^{2}+80 b^{3}+65 b^{4}+36 b^{5}+9 b^{6}\right)(1+\epsilon)
\end{aligned}
$$

\section{APPENDIX C: BULK STRESS COEFFICIENTS IN A SECOND-ORDER FLUID MATRIX}

The bulk stress coefficients $\Sigma_{0}$ and $\Sigma_{1}$ found in Eq. (57) are

$$
\begin{aligned}
& \Sigma_{0}=4 \epsilon\left[1+\frac{10 b^{3}\left(1+b+b^{2}+b^{3}+b^{4}+b^{5}+b^{6}\right) \phi}{(b-1)^{3}\left(4+16 b+40 b^{2}+55 b^{3}+40 b^{4}+16 b^{5}+4 b^{6}\right)}\right], \\
& \Sigma_{1}=4(1+\epsilon)+\frac{\Sigma_{\phi} \phi}{7(b-1)^{4}\left(4+16 b+40 b^{2}+55 b^{3}+40 b^{4}+16 b^{5}+4 b^{6}\right)^{3}},
\end{aligned}
$$

for the coefficient of solid volume fraction

$$
\begin{aligned}
\Sigma_{\phi}= & 60 b^{3}\left(-32-256 b-1152 b^{2}-3140 b^{3}-4960 b^{4}-3336 b^{5}+4802 b^{6}+18964 b^{7}\right. \\
& +34140 b^{8}+45760 b^{9}+53768 b^{10}+59004 b^{11}+59508 b^{12}+52185 b^{13}+37320 b^{14} \\
& \left.+20800 b^{15}+8900 b^{16}+2880 b^{17}+640 b^{18}+80 b^{19}\right)+120 b^{3}\left(-8-64 b-288 b^{2}\right. \\
& -660 b^{3}-240 b^{4}+2896 b^{5}+10353 b^{6}+21436 b^{7}+32580 b^{8}+40280 b^{9}+43712 b^{10} \\
& +43236 b^{11}+38812 b^{12}+30660 b^{13}+20320 b^{14}+10800 b^{15}+4500 b^{16} \\
& \left.+1440 b^{17}+320 b^{18}+40 b^{19}\right) \epsilon .
\end{aligned}
$$

\section{APPENDIX D: EXTRA BULK STRESS CONTRIBUTIONS FROM THE MEAN POLYMER STRESS AND STRESSLET TERMS}

The bulk extra stress contributions come from both the mean polymer stress and the stresslet as given in Eqs. (24) and (21), respectively. The mean polymer stress terms are

$$
\begin{aligned}
4 \epsilon\left\langle\widehat{E_{i k}^{\prime} \Omega_{k j}^{\prime}}\right\rangle & =-\frac{60 b^{3}\left(1+b+b^{2}+b^{3}+b^{4}+b^{5}+b^{6}\right) \epsilon \phi}{7(b-1)^{3}\left(4+16 b+40 b^{2}+55 b^{3}+40 b^{4}+16 b^{5}+4 b^{6}\right)} \widehat{E_{i k}^{\infty} E_{k j}^{\infty},} \\
4(1+\epsilon)\left\langle\widehat{E_{i k}^{\prime} E_{k j}^{\prime}}\right\rangle & =\frac{20 b^{3}\left(1+b+b^{2}+b^{3}+b^{4}+b^{5}+b^{6}\right)(1+\epsilon) \phi}{(b-1)^{3}\left(4+16 b+40 b^{2}+55 b^{3}+40 b^{4}+16 b^{5}+4 b^{6}\right)} \widehat{E_{i k}^{\infty} E_{k j}^{\infty},}
\end{aligned}
$$


which introduces the relationship $\left\langle\widehat{\mathbf{E}^{\prime} \cdot \mathbf{\Omega}^{\prime}}\right\rangle=-3\left\langle\widehat{\mathbf{E}^{\prime} \cdot \mathbf{E}^{\prime}}\right\rangle / 7$ using the ensemble averaging process and cell model as detailed in Sec. II. The stresslet term is

$$
\begin{aligned}
\frac{3 \phi}{4 \pi} \widehat{S}_{i j}^{1}= & \frac{40 b^{3}\left(1+b+b^{2}+b^{3}+b^{4}+b^{5}+b^{6}\right) \epsilon \phi}{(b-1)^{3}\left(4+16 b+40 b^{2}+55 b^{3}+40 b^{4}+16 b^{5}+4 b^{6}\right)} \widehat{E_{i k}^{\infty} \Omega_{k j}^{\infty}} \\
& +\frac{S_{\phi} \phi}{7(b-1)^{4}\left(4+16 b+40 b^{2}+55 b^{3}+40 b^{4}+16 b^{5}+4 b^{6}\right)^{3}} \widehat{E_{i k}^{\infty} E_{k j}^{\infty}}
\end{aligned}
$$

for the coefficient

$$
\begin{aligned}
S_{\phi}= & 20 b^{3}\left(16+128 b+576 b^{2}+2620 b^{3}+10880 b^{4}+30648 b^{5}+61789 b^{6}+97436 b^{7}\right. \\
& +127284 b^{8}+145288 b^{9}+153296 b^{10}+152148 b^{11}+137980 b^{12}+109172 b^{13}+71304 b^{14} \\
& \left.+36640 b^{15}+14660 b^{16}+4608 b^{17}+1024 b^{18}+128 b^{19}\right)+40 b^{3}\left(8+64 b+288 b^{2}\right. \\
& +1460 b^{3}+6640 b^{4}+20304 b^{5}+44597 b^{6}+75892 b^{7}+104844 b^{8}+123128 b^{9} \\
& +128848 b^{10}+122604 b^{11}+104852 b^{12}+78442 b^{13}+49344 b^{14}+25040 b^{15} \\
& \left.+10060 b^{16}+3168 b^{17}+704 b^{18}+88 b^{19}\right) \epsilon .
\end{aligned}
$$

[1] L. Bergström, Shear thinning and shear thickening of concentrated ceramic suspensions, Colloids Surf. A 133, 151 (1998).

[2] A. J. Liu and S. R. Nagel, Jamming and Rheology: Constrained Dynamics on Microscopic and Macroscopic Scales (CRC Press, Boca Raton, FL, 2001).

[3] N. Fernandez, R. Mani, D. Rinaldi, D. Kadau, M. Mosquet, H. Lombois-Burger, J. Cayer-Barrioz, H. J. Herrmann, N. D. Spencer, and L. Isa, Microscopic Mechanism for Shear Thickening of Non-Brownian Suspensions, Phys. Rev. Lett. 111, 108301 (2013).

[4] M. Wyart and M. E. Cates, Discontinuous Shear Thickening Without Inertia in Dense Non-Brownian Suspensions, Phys. Rev. Lett. 112, 98302 (2014).

[5] R. Seto, G. G. Giusteri, and A. Martiniello, Microstructure and thickening of dense suspensions under extensional and shear flows, J. Fluid Mech. 825, R3 (2017).

[6] X. Xu, S. A. Rice, and A. R. Dinner, Relation between ordering and shear thinning in colloidal suspensions, Proc. Natl. Acad. Sci. USA 110, 1 (2013).

[7] A. Vázquez-Quesada, A. Mahmud, S. Dai, M. Ellero, and R. I. Tanner, Investigating the causes of shearthinning in non-colloidal suspensions: Experiments and simulations, J. Non-Newtonian Fluid Mech. 248, 1 (2017).

[8] D. J. Highgate and R. W. Whorlow, Rheological properties of suspensions of spheres in non-Newtonian media, Rheol. Acta 9, 569 (1970).

[9] T. Kataoka, T. Kitano, M. Sasahara, and K. Nishijima, Viscosity of particle filled polymer melts, Rheol. Acta 17, 149 (1978).

[10] H. A. Barnes, A review of the rheology of filled viscoelastic systems, Rheol. Rev. 2003, 1 (2003).

[11] M. Liard, N. S. Martys, W. L. George, D. Lootens, and P. Hebraud, Scaling laws for the flow of generalized Newtonian colloidal suspensions, J. Rheol. 58, 1993 (2014).

[12] S. Mall-Gleissle, W. Gleissle, G. H. McKinley, and H. Buggisch, The normal stress behavior of suspensions with viscoelastic matrix fluids, Rheol. Acta 41, 61 (2002).

[13] R. Tanner, S. Dai, F. Qi, and K. Housiadas, Viscometric functions of semidilute noncolloidal suspensions of spheres in a viscoelastic matrix, J. Non-Newtonian Fluid Mech. 201, 130 (2013).

[14] R. Tanner, F. Qi, and S. Dai, Scaling the normal stresses in concentrated non-colloidal suspensions of spheres, Rheol. Acta 52, 291 (2013).

[15] S. Dai, F. Qi, and R. I. Tanner, Viscometric functions of concentrated non-colloidal suspensions of spheres in a viscoelastic matrix, J. Rheol. 58, 183 (2014). 
[16] D. L. Koch, E. F. Lee, and I. Mustafa, Stress in a dilute suspension of spheres in a dilute polymer solution subject to simple shear flow at finite Deborah numbers, Phys. Rev. Fluids 1, 013301 (2016).

[17] S. Krishnan, E. S. G. Shaqfeh, and G. Iaccarino, Fully resolved viscoelastic particulate simulations using unstructured grids, J. Comput. Phys. 338, 313 (2017).

[18] A. Einstein, Eine neue bestimmung der moleküldimensionen, Annalen der Physik 324, 289 (1906).

[19] G. K. Batchelor and J. T. Green, The determination of the bulk stress in a suspension of spherical particles to order c $\mathrm{c}^{2}$, J. Fluid Mech. 56, 401 (1972).

[20] M. Cohen and D. Turnbull, Molecular transport in liquids and glasses, J. Chem. Phys. 31, 1164 (1959).

[21] Z. Cheng, J. Zhu, P. M. Chaikin, S. E. Phan, and W. B. Russel, Nature of the divergence in low shear viscosity of colloidal hard-sphere dispersions, Phys. Rev. E 65, 041405 (2002).

[22] I. M. Krieger and T. J. Dougherty, A mechanism for non-Newtonian flow in suspensions of rigid spheres, Trans. Soc. Rheol. 3, 137 (1959).

[23] P. N. Kaloni and V. Statsna, Steady-shear rheological behavior of the suspension of spherical particles in a second-order fluid, Polym. Eng. Sci. 23, 465 (1983).

[24] K. Sun and K. Jayamaran, Bulk rheology of dilute suspensions in viscoelastic liquids, Rheol. Acta 23, 84 (1984).

[25] R. T. Mifflin, Dissipation in a dilute system of spheres in a second-order fluid, J. Non-Newtonian Fluid Mech. 17, 267 (1985).

[26] N. A. Patankar and H. H. Hu, Rheology of a suspension of particles in viscoelastic fluids, J. NonNewtonian Fluid Mech. 96, 427 (2001).

[27] F. Greco, G. D'Avino, and P. L. Maffettone, Rheology of a dilute suspension of rigid spheres in a secondorder fluid, J. Non-Newtonian Fluid Mech. 147, 1 (2007).

[28] K. D. Housiadas and R. I. Tanner, On the rheology of a dilute suspension of rigid spheres in a weakly viscoelastic matrix fluid, J. Non-Newtonian Fluid Mech. 162, 88 (2009).

[29] D. L. Koch and G. Subramanian, Corrigendum to "The stress in a dilute suspension of spheres suspended in a second-order fluid subject to a linear velocity field," J. Non-Newtonian Fluid Mech. 153, 202 (2008).

[30] D. L. Koch and G. Subramanian, The stress in a dilute suspension of spheres suspended in a second-order fluid subject to a linear velocity field, J. Non-Newtonian Fluid Mech. 138, 87 (2006).

[31] J. M. Rallison, The stress in a dilute suspension of liquid spheres in a second-order fluid, J. Fluid Mech. 693, 500 (2012).

[32] R. Simha, A treatment of the viscosity of concentrated suspensions, J. Appl. Phys. 23, 1020 (1952).

[33] J. Happel, Viscosity of suspensions of uniform spheres, J. Appl. Phys. 28, 1288 (1957).

[34] H. Ohshima, Sedimentation potential in a concentrated suspension of spherical colloidal particles, J. Colloid Interface Sci. 208, 295 (1998).

[35] J. M. Ding and H. J. Keh, The electrophoretic mobility and electric conductivity of a concentrated suspension of colloidal spheres with arbitrary double-layer thickness, J. Colloid Interface Sci. 236, 180 (2001).

[36] R. Pal, Evaluation of theoretical viscosity models for concentrated emulsions at low capillary numbers, Chem. Eng. J. 81, 15 (2001).

[37] H. Usui, Prediction of dispersion characteristics and rheology in dense slurries, J. Chem. Eng. Jpn. 35, 815 (2002).

[38] M. Yang and E. S. G. Shaqfeh, Mechanism of shear thickening in suspensions of rigid spheres in Boger fluids. Part I: Dilute suspensions, J. Rheol. 62, 1363 (2018).

[39] J. Einarsson, M. Yang, and E. S. G. Shaqfeh, The Einstein viscosity with fluid elasticity, Phys. Rev. Fluids 3, 013301 (2017).

[40] M. S. Chong, A. E. Perry, B. J. Cantwell, M. S. Chong, and A. E. Perry, A general classification of three-dimensional flow fields, Phys. Fluids A 2, 765 (1990).

[41] J. D. Sherwood, Cell models for suspension viscosity, Chem. Eng. Sci. 61, 6727 (2006).

[42] R. S. Rivlin and J. L. Ericksen, in Stress-Deformation Relations for Isotropic Materials, edited by G. I. Barenblatt, D. D. Joseph (Springer, New York, 1997).

[43] M. Yang and E. S. G. Shaqfeh, Mechanism of shear thickening in suspensions of rigid spheres in Boger fluids. Part II: Suspensions at finite concentration, J. Rheol. 62, 1379 (2018). 
[44] R. J. Phillips and L. Talini, Chaining of weakly interacting particles suspended in viscoelastic fluids, J. Non-Newtonian Fluid Mech. 147, 175 (2007).

[45] J. H. Peery, Fluid mechanics of rigid and deformable particles in shear flows at low Reynolds numbers, Ph.D. thesis, Princeton University (1966).

[46] B. P. Ho and L. G. Leal, Migration of rigid spheres in a two-dimensional unidirectional shear flow of a second-order fluid, J. Fluid Mech. 76, 783 (1976).

[47] M. Yang, S. Krishnan, and E. S. G. Shaqfeh, Numerical simulations of the rheology of suspensions of rigid spheres at low volume fraction in a viscoelastic fluid under shear, J. Non-Newtonian Fluid Mech. 234, 51 (2016).

[48] D. Park, Direct calculation of inter-particle distance in suspension by image processing, Powder Technol. 330, 252 (2018). 